\title{
Fine-particle defluidization: Interaction between cohesion, Young's modulus and static bed height
}

\author{
Peiyuan Liu, Casey Q. LaMarche, Kevin M. Kellogg, and Christine M. Hrenya* \\ Department of Chemical and Biological Engineering, University of Colorado Boulder, Boulder, CO \\ 80309, USA
}

\begin{abstract}
The defluidization of fine (mildly cohesive) glass particles with mean size $69 \mu \mathrm{m}$ is studied by DEMCFD simulations and experiments for quantitative model validation. Cohesion arising from van der Waals forces between particles is explicitly incorporated into DEM-CFD, along with the corresponding effects of surface roughness. Due to computational limitations, DEM-CFD simulations often use artificially soft particles and systems much smaller than experiments, making the direct comparison between simulation and experimental results questionable. This computational obstacle was recently overcome for coarse (non-cohesive) particles via the identification of a system-size-independent measurement, thereby allowing for the direct comparison between small-scale simulations and largescale experiments (LaMarche et al., 2015b). Here, we assess the applicability of the same measurement, namely the defluidization curve, to cohesive systems. Unlike non-cohesive systems, the simulation results exhibit a sensitivity to Young's modulus and (low) static bed heights. These observations are explained by the enhanced cohesive effect at lower Young's modulus and decreasing bed porosity with increasing static bed height. Nonetheless, by using the true material Young's modulus in the DEM-CFD simulations and sufficiently large static bed height, a system-size-independent defluidization measurement is achieved that compares well with experimental data from a much larger system. This ability to directly compare small-scale simulations and large-scale experiments via a system-size independent measurement (defluidization) allows for the quantitative validation of the particle-level models in DEM-CFD without resorting to particle-level measurements and/or unrealistically small experimental systems.

\footnotetext{
${ }^{*}$ Corresponding author.

E-mail address: hrenya@colorado.edu
} 
Keywords: DEM-CFD; fluidized beds; fine particles; cohesion

\section{Introduction}

Fluidized beds are widely used in industry due to high rates of mass and heat transfer (Kunii and Levenspiel, 1991). Depending on the size and density of particles, different fluidization behaviors are observed in air-fluidized beds operated at ambient pressure and temperature (Geldart, 1973). For example, "coarse" glass particles with size $>100 \mu \mathrm{m}$ show immediate bubbling when the gas velocity reaches the minimum fluidization velocity $U_{m f}$, at which the solid phase is fully supported by the gas

phase. For "fine" particles with size $<100 \mu \mathrm{m}$, a non-bubbling (homogenous) fluidization emerges for gas velocities between $U_{m f}$ and a minimum bubbling velocity $U_{m b}$ (Valverde et al., 2003). Fine particles also have pronounced hysteresis in fluidization-defluidization curves (Rietema and Piepers, 1990; Tsinontides and Jackson, 1993; Ojha et al., 2000). As particle size continues to decrease below $20 \mu \mathrm{m}$, these extra-fine particles usually show non-fluidization by forming channels and cracks throughout the bed with little mixing.

An important factor responsible for the different fluidization behaviors (Castellanos et al., 1999) is the changing impact of interparticle cohesion resulting from the van der Waals force with varying particle size. The effects of cohesion on granular systems in general have piqued interest from both physicists and engineers. Novel properties of granular matter arise from cohesion, such as increased mechanical strength (Halsey and Levine, 1998), agglomerates formation (Weber et al., 2004; Royer et al., 2009) and avalanching flow (Quintanilla et al., 2001). Cohesion also has complicated impact on granular segregation (Geromichalos et al., 2003; Li and McCarthy, 2003) and inclination angle of particles in rotating drums (Xu et al., 2007). The ratio of the cohesion to the weight of a single particle, often referred as the Bond number Bo, can be used to evaluate the impact of cohesion. Since the van der Waals force is proportional to the particle diameter for perfectly smooth particles (or radius of curvature of asperities for rough particles (Massimilla and Donsi, 1976)) while particle weight is proportional to the cube of the particle diameter, Bo decreases sharply with increasing particle diameter (Sundaresan, 2003; Castellanos, 2005). The transition of fluidization regimes with decreasing particle diameter was linked with the corresponding change of Bo (Valverde and Castellanos, 2007). For coarse particles, Bo $<<1$ and the effect of cohesion is largely negligible. For fine particles, which is the focus of this work, 
the van der Waals force and particle weight are of comparable magnitude (Bo $\sim 1)$ and significant cohesive effects on fluidization are expected.

A commonly-used method to study fluidization is the coupled Discrete Element Method-Computational Fluid Dynamics (DEM-CFD), where DEM refers to the solution of trajectories of each solid particle by explicit integration of Newton's equations of motion and CFD refers to the conventional Navier-Stokes solution for the gas phase (Tsuji et al., 1992; van der Hoef et al., 2008). Generally speaking, compared with the Two-Fluid Models (TFM), which treats gas and solid phases as interacting continua, DEMCFD does not require closures for solid phase stress and can be used to drive the development of TFM by highlighting important solid properties to consider in TFM (Goldschmidt et al., 2001) or providing validation data for TFM incorporated with new physics (Murray et al., 2012; Galvin and Benyahia, 2014).

With specific regard to cohesion, DEM-CFD enables a more straightforward incorporation of cohesion than TFM. The challenges with the TFM include modifying the kinetic-theory-based closure for solid stress and tracking of the evolution of agglomerates size distribution. Progress has been made to address these issues via a number of routes: "cohesive kinetic theory" (Ding and Gidaspow, 1994; Kim and Arastoopour, 2002); force balance on agglomerates (Motlagh et al., 2014); and population balance models (Fan et al., 2004). However, the application of these models are limited either by their simplifying treatments, such as assuming a constant probability of agglomeration (Fan et al., 2004), or by not allowing for deagglomeration (Kim and Arastoopour, 2002). Unlike TFM, DEM-CFD solves the force balance for each particle so that particle-particle cohesion can be added explicitly. Due to this capability, DEM has become a popular tool to study cohesive granular systems. Studies focusing on both statics and dynamics of cohesive particles are widely available in literature, with a recent review in ref. (Guo and Curtis, 2015).

Despite the broad application of DEM-CFD to study cohesive particles (Mikami et al., 1998; Rhodes et al., 2001; Pandit et al., 2006; Weber and Hrenya, 2006, 2007; Hou et al., 2012; Galvin and Benyahia, 2014), quantitative validation of simulation results by comparison with experiments is rarely conducted due to high computational overhead. In particular, since particle collisions need a small time step to resolve, DEM-CFD simulations are computationally intensive and it is essentially impossible to simulate typical, lab-scale experiments. The situation worsens for smaller particles, since the time step is 
proportional to particle size. For example, our recent study on fine particles (Liu and Hrenya, 2014) showed that even for a pseudo-2D fluidized bed (bed with thickness of 1 particle diameter), the bed width attainable by DEM-CFD is limited to $\sim 1 \mathrm{~cm}$, much smaller than typical experiments with bed width and thickness $>10 \mathrm{~cm}$. To overcome this computational challenge, it is desirable to increase the simulation time step or have a scale-independent parameter to compare with experiments. While these options are viable for non-cohesive particles, as summarized below, their applicability to cohesive systems remains unclear.

To increase the time step used in DEM simulations, a common treatment is to use a Young's modulus of the particle that is smaller than true material value (i.e., "softer" particles). Decreasing Young's modulus increases the collision duration, thereby allowing for a larger time step. For bubbling beds of noncohesive particles, DEM-CFD results are found to be insensitive to Young's modulus and thus using an artificially small Young's modulus results in satisfactory agreement with experiments (Mikami et al., 1998; Di Maio and Di Renzo, 2004; Muller et al., 2008). The main reason for the insensitivity lies in the increasing importance of gas-solid interaction inside bubbling bed (Tsuji et al., 1993; Mikami et al., 1998; Di Maio and Di Renzo, 2004) compared with cohesive particle interactions, so that the artifact associated with the overestimation of collision duration (for soft particles) becomes secondary. However, for cohesive particles, sensitivity of DEM-CFD results to Young's modulus has been observed. Moreno-Atanasio et al. (2007) varied the spring constant in DEM-CFD simulations of fluidized bed. (Similar to the Young's modulus in a Hertzian contact model, the spring constant is a measure of material stiffness in linear contact model). They found that the effect of the spring constant becomes more evident with increasing cohesion, and that cohesive particles could transit from nonfluidization to full fluidization by merely increasing the spring constant. Kobayashi et al. (2013) recently found that the bubble size in fluidized bed increases with spring constant in DEM-CFD simulations, which was ascribed to the decreasing critical deagglomeration velocity $v_{\text {crit }}$, the velocity below which two particles agglomerate after impact, with increasing spring constants. By rescaling the cohesion with the spring constant while keeping $v_{c r i t}$ equal to the value predicted using the realistic spring constant, they proposed a "dynamic adhesion force model", which allows for using lower spring constants in bubbling simulations for cohesive particles. They conducted experiments in a small fluidized bed (18 $\mathrm{mm} \times 3.6 \mathrm{~mm}$ ) with the same geometry as simulations and observed qualitative agreement. 
Another approach for a meaningful comparison between DEM-CFD and experiments is the identification of a measurement that is independent of system size. Our recent study (LaMarche et al., 2015b) found that for non-cohesive particles, defluidization curves are insensitive to system scale and large-scale experimental measurement can be directly compared with small-scale simulations for purposes of model validation. For cohesive particles, however, it is unclear if defluidization remains independent of system size.

In this work, DEM-CFD simulations and experiments were conducted to study the defluidization of fine glass particles. The surface roughness of particles used in experiments was measured experimentally, and a cohesion model that incorporates surface roughness (LaMarche et al., 2015a) was used in DEMCFD. The defluidization curves, i.e., the bed pressure drop as a function of decreasing superficial gas velocity, were collected. Two critical questions motivated this work: (i) does Young's modulus used in DEM simulations affect the defluidization of cohesive particles, and (ii) is cohesive defluidization independent of system size? Our results indicate that in contrast to non-cohesive particles, Young's modulus and static bed height both play a role in cohesive defluidization, but comparing small-scale simulation with large-scale experiments is still feasible if realistic Young's modulus and a sufficient static bed height are used in DEM-CFD. The mechanisms for the different observations between noncohesive and cohesive particles are also discussed.

\section{Experiment}

\subsection{Fluidized bed}

The experimental fluidization setup is detailed in prior studies (Chew et al., 2010). The fluidized bed consists of a Plexiglas cylinder with diameter $D_{\exp }=18.4 \mathrm{~cm}$, as shown in Fig. 1 . The air is distributed to the bottom of the column using a sintered stainless steel porous distributor plate. The pore size of the porous plate is $5 \mu \mathrm{m}$, ensuring uniform flow distribution of gas as the pressure drop across the distributor was always greater than $10 \%$ of the pressure drop across the bed at minimum fluidization velocity (Kunii and Levenspiel, 1991). A sufficiently high initial gas velocity ensures that the bed remains bubbling after several velocity reductions during defluidization. The bed is held at this high initial gas velocity for 15 to 20 minutes, after the relative humidity of the system drops below 5\%, and

prior to reducing the gas velocity. Each gas velocity was held constant for 60 seconds to ensure a 
pressure drop across the bed had reached a (statistically) steady state. Pressure drop and gas velocity measurements were collected at $60 \mathrm{~Hz}$. Five trials were conducted.

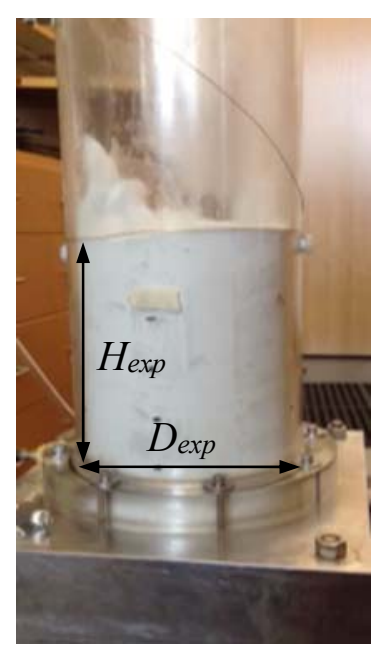

Fig. 1. Fluidized bed used in experiments.

The relative humidity was controlled using a packed silica dryer at the inlet of the fluidized bed and the relative humidity was held below $10 \%$ for all experiments to avoid adhesion caused by capillary forces (LaMarche et al., 2015c). For the particles studied here, the defluidization curves were not sensitive to the relative humidity when compared to experiments performed with relative humidity measured at $0.5 \%$ (when the relative humidity is below $3 \%$, the measurement accuracy diminishes), indicating it is valid to assume humidity is not affecting the results. To minimize static buildup on the particles, antistatic spray (Kensington Dust Guardian) was applied to the walls of the column and a copper wire and metal base were used to ground the system.

\subsection{Materials}

The spherical soda-lime glass particles studied are monodisperse of diameter of $69 \mu \mathrm{m} .6 \mathrm{~kg}$ particles were used in experiments, corresponding to a static bed height $H_{\text {exp }}=16.1 \mathrm{~cm}$. To measure the sliding friction coefficient, multiple sleds, consisting of four glass particles epoxied to the bottom of a thin glass square, are produced and tracked with a high-speed camera as they slide down an angled soda-lime glass plate (LaMarche et al., 2015c). The particles are fixed to the sled in order to isolate the sliding friction. The entire device is contained in a chamber, which allows for controlling the relative humidity to within 
the range used for the fluidization experiments. The measured sled accelerations are used to calculate the sliding friction coefficient of $\mu=0.273 \pm 0.02$. The normal coefficient of restitution $e_{n}=0.97$ was reported in the literature for glass particles (Foerster et al., 1994).

\section{Numerical method}

The open-source code MFIX (Multiphase Flow with Interphase eXchange) (Syamlal et al., 1993) was used in this work to conduct the DEM-CFD simulations. Below the governing equations of DEM-CFD are briefly described. Details of the model implementation and verification can be found in ref. (Garg et al., 2012).

\subsection{DEM-CFD governing equations}

In DEM-CFD simulations, the solids phase is treated as discrete particles. For one particle of mass $m$, its transitional velocity $\boldsymbol{u}_{s}$ and rotational velocity $\boldsymbol{\omega}_{s}$ are solved by Newton's equation of motion

$$
\begin{aligned}
& m \frac{d \mathbf{u}_{s}}{d t}=\boldsymbol{f}_{c}+\boldsymbol{f}_{f}+\boldsymbol{f}_{v d W}+m \mathbf{g} \\
& I \frac{d \boldsymbol{\omega}_{s}}{d t}=\boldsymbol{T}
\end{aligned}
$$

where $\boldsymbol{f}_{c}, \boldsymbol{f}_{f}$, and $\boldsymbol{f}_{v d W}$ are, respectively, the contact force due to particle-particle and particle-wall contacts, particle-fluid drag force and van der Waals force; $\mathbf{g}$ is the acceleration due to gravity; $\boldsymbol{T}$ is the torque due to the tangential component of the contact force; and $\boldsymbol{I}$ is the moment of inertia. The contact force $f_{c}$ between particle $i$ and $j$ is given by the visco-elastic contact model in normal and tangential directions (Tsuji et al., 1992). The contact force in the normal direction $f_{c}^{n}$ is given by

$$
f_{c}^{n}=\frac{4 E_{e f f} \sqrt{R_{e f f}}}{3} \delta_{n}^{\frac{3}{2}}+\eta_{n} \frac{d \delta_{n}}{d t}
$$

where $1 / E_{\text {eff }}=\left(1-v_{i}^{2}\right) / E_{i}+\left(1-v_{j}^{2}\right) / E_{j},(k=i, j), E$ is Young's modulus, $v$ is the Poisson's ratio, $R_{\text {eff }}=R_{i} R_{j} /\left(R_{i}+R_{j}\right)$ is the effective radius and $\delta_{n}$ is the normal geometric overlap between two 
particles. $f_{c}^{n}$ in Eq.(3) is a sum of two terms. The first term represents the repulsion from the elastic deformation during physical contact of two particles, which is given by the Hertz contact model (Johnson, 1985). The second term is a dashpot damping force which accounts for the dissipation of mechanical energy during particle collision. By solving the equations of motion for a binary particle collision without cohesion, the normal damping coefficient $\eta_{n}$ is related analytically to the normal coefficient of restitution $e_{n}$ by Antypov and Elliott (2011).

$$
\eta_{n}=\frac{-2 \sqrt{15} R_{e f f}^{\frac{1}{4}} \sqrt{m_{e f f} E_{e f f}} \ln e_{n}}{3 \sqrt{\pi^{2}+\ln ^{2} e_{n}}} \delta_{n}^{\frac{1}{4}}
$$

The contact force in the tangential direction $f_{c}^{t}$ is given by Tsuji et al. (1992)

$$
f_{c}^{t}= \begin{cases}\frac{16 G_{\text {eff }} \sqrt{R_{\text {eff }}}}{3} \delta_{n}^{\frac{1}{2}} \delta_{t}+\eta_{t} \frac{d \delta_{t}}{d t} & f_{c}^{t}<\mu f_{c}^{n} \\ \mu f_{c}^{n} & f_{c}^{t} \geq \mu f_{c}^{n}\end{cases}
$$

where $G_{k}=E_{k} /\left(2+2 v_{k}\right),(k=i, j)$ is shear modulus and $\delta_{t}$ is the tangential displacement during particle collision. The tangential damping coefficient $\eta_{t}$ is assumed to be equal to $\eta_{n}$ in this work, similar to the treatment by Tsuji et al. (1992). A finite Coulomb sliding friction between two particles is turned on whenever $f_{c}^{t}$ exceeds that maximum static friction approximated to be $\mu f_{c}^{n}$, where $\mu$ is the sliding friction coefficient. Particle-wall contacts are treated analogously with particle-particle contacts.

The fluid-particle interaction $\boldsymbol{f}_{f}$ associated with particle $i$ is given by

$$
\boldsymbol{f}_{f}^{i}=-V_{i} \nabla P_{g}-\frac{V_{i} \beta}{1-\varepsilon}\left(\mathbf{u}_{g}-\mathbf{u}_{s}\right)
$$

where $V_{i}$ is the volume of the particle, $P_{g}$ is the gas pressure, $\beta$ is the gas-solid frictional coefficient, $\boldsymbol{u}_{g}$ is the velocity of gas, and $\varepsilon$ is the porosity. $\beta$ can be obtained from different drag models. Detailed expressions of $\beta$ used in this work can be found in Benyahia et al. (2006), who extended the drag model by Hill et al. (2001a, b) so that it can be applied for the range of porosity and Reynolds number typical of fluidized bed. Our recent work (LaMarche et al., 2015b) shows excellent quantitative agreement 
achieved in terms of defluidization curves for the non-cohesive $300 \mu \mathrm{m}$ and $151 \mu \mathrm{m}$ particles based the above drag model. Following our recent work (LaMarche et al., 2015b), particle diameter in the expression of the gas-solid friction coefficient $\beta$ is multiplied by the particle sphericity measured experimentally to account for the shape effect of particles in gas-solid drag. Considering particle sphericity in simulations was found essential for model validation with non-cohesive particles. Details about the measurement of particle sphericity can be found in ref. (LaMarche et al., 2015b).

The fluid-phase governing equations are given by

$$
\begin{aligned}
& \frac{\partial \varepsilon \rho_{g}}{\partial t}+\nabla \cdot\left(\varepsilon \rho_{g} \mathbf{u}_{g}\right)=0 \\
& \frac{\partial\left(\varepsilon \rho_{g} \mathbf{u}_{g}\right)}{\partial t}+\nabla \cdot\left(\varepsilon \rho_{g} \mathbf{u}_{g} \mathbf{u}_{g}\right)=-\varepsilon \nabla P_{g}+\nabla \cdot \tau_{g}+\varepsilon \rho_{g} \mathbf{g}-\mathbf{I}_{g s}
\end{aligned}
$$

where $\boldsymbol{\tau}_{g}$ is the gas shear stress tensor and $\mathbf{I}_{\mathrm{gs}}$ is the gas-solid momentum transfer, given by

$$
\mathbf{I}_{g s}=\frac{1}{V_{m}} \sum_{i=1}^{N_{m}} \frac{\phi_{i}^{m} V_{i} \beta}{1-\varepsilon}\left(\mathbf{u}_{i}-\mathbf{u}_{g}\right)
$$

where $V_{m}$ is the volume of a CFD cell $m$ and $N_{m}$ is the number of particles in the cell. The interpolation

factor $\phi$ determines the contribution of the drag force of each particle to the cell, which is inversely proportional to the distance from particle location to the cell center. The gas phase is treated as incompressible and more details about the fluid solver, which is based on the control volume formulation of Patankar (1980), can be found in the theory manual of MFIX (Syamlal et al., 1993).

\subsection{Cohesion model}

Cohesion arising from van der Waals forces is incorporated in DEM by extending the Rabinovich model for cohesion between a rough particle and a smooth wall (Rabinovich et al., 2000a, b) to cohesion between two rough particles (LaMarche et al., 2015a), as shown in Fig. 2. In this model, roughness is assumed to cover the entire surface of the sphere. The roughness is modeled via a superposition of two waves with small-scale and large-scale wavelengths, $\lambda_{i s}$ and $\lambda_{i L}$ in Fig. 2 . Both waves take the shape of alternating spherical caps with internal radius (asperity radius $r_{i S}$ and $r_{i L}$ in Fig. 2) related to rms amplitudes and wavelengths of respective scales. Further, as also illustrated in Fig. 2, the model assumes 
that the peak on the roughness of particle $i$ is always in line with the peak on the roughness of particle $j$, i.e. peak-to-peak contact. The van der Waals force between two particles is calculated by a pair-wise summation of the cohesion between primary particles and asperities related to two scales of surface roughness, given by

$$
\begin{aligned}
& f_{v d W}= \frac{A R_{i} R_{j}}{6\left(R_{i}+R_{j}\right)\left(D+y_{i L}+y_{i S}+y_{j L}+y_{j S}\right)^{2}}+\frac{A R_{i} r_{j L}}{6\left(R_{i}+r_{j L}\right)\left(D+y_{i L}+y_{i S}+y_{j S}\right)^{2}}+ \\
& \frac{A R_{i} r_{j S}}{6\left(R_{i}+r_{j S}\right)\left(D+y_{i L}+y_{i S}\right)^{2}}+\frac{A r_{i L} R_{j}}{6\left(r_{i L}+R_{j}\right)\left(D+y_{i S}+y_{j L}+y_{j S}\right)^{2}}+ \\
& \frac{A r_{i S} R_{j}}{6\left(r_{i S}+R_{j}\right)\left(D+y_{i L}+y_{j S}\right)^{2}}+\frac{A r_{i L} r_{j L}}{6\left(r_{i L}+r_{j L}\right)\left(D+y_{i S}+y_{j S}\right)^{2}}+ \\
& \frac{A r_{i L} r_{j S}}{6\left(r_{i L}+r_{j S}\right)\left(D+y_{i S}\right)^{2}}+\frac{A r_{i S} r_{j L}}{6\left(r_{i S}+r_{j L}\right)\left(D+y_{j S}\right)^{2}}+\frac{A r_{i S}}{6\left(r_{i S}+r_{j S}\right) D^{2}} \\
& r_{k l}=\frac{\lambda_{k l}^{2}}{32 k_{1} r m s_{k l}}, y_{k l}=k_{1} r m s_{k l} \quad(k=i, j \text { and } l=L, S)
\end{aligned}
$$

where $k_{1}=1.817$ is a physically-based constant that relates rms roughness and the maximum peak height $y_{\max }$ (see. Eq. (18) in ref. (Rabinovich et al., 2000a)). $A$ is the Hamaker constant and $D$ is the separation distance between two asperities. $r m s$ is the root mean square roughness and $\lambda$ is the peak-to-peak distance as defined in the original model (Rabinovich et al., 2000a). Subscripts $L$ and $S$ represent largeand small-scale roughness parameters, respectively. When $D$ is less than the intermolecular distance $D_{0}$ $=0.3 \mathrm{~nm}$ (Israelachvili, 2011), the cohesion is set to the constant value $F_{v d W}\left(D_{0}\right)$. On the right hand side of Eq. (10), the first term refers to cohesive forces between the two (smooth portions of) particles, the next two terms refer to cohesion between particle $i$ and the large and small-scale asperities on $j$, respectively. The 4th and 5th terms refer to the cohesion between particle $j$ and the large and small-scale asperities on $i$, respectively. The 6th and 7th terms refer to the cohesion between large scale asperity of particle $i$ and the large and small-scale asperities on $j$, respectively. The 8th and 9th terms refer to the cohesion between small scale asperity of particle $i$ and the large and small-scale asperities on $j$, respectively. rms and $\lambda$ for both large- and small-scale roughness were obtained from the analysis of atomic force microscopy (AFM) measurements of particle surface. More details on the determination of $r m s$ and $\lambda$ can be found in ref. (LaMarche et al., 2015a), where agreement was observed between the prediction of Eq. (10) and the measurement for the soda lime particles used in our fluidized bed 
experiments. Thus, incorporation of the cohesion model in Eq. (10) in DEM enables the validation of simulation results by comparison with experiments. However, it is worth noting that the qualitative findings of this work, to be discussed in the results section, are not affected by the type of cohesion model used. For example, we found that the sensitivity of simulation results to bed height can be captured via a simpler cohesion model, the Hamaker model (Hamaker, 1937) (results not shown).

Particle-wall cohesion was not incorporated since side walls are treated as non-cohesive to reduce wall effects (as detailed in following section), and experiments show negligible cohesion between particles and the distributor plate (i.e., pressure drop during complete fluidization equals bed weight divided by cross-sectional area). The asperities on particle surfaces are considered to have the same material properties as the particle, and the contact force between particles is turned on in simulations when two asperities are overlapping.

Large scale roughness

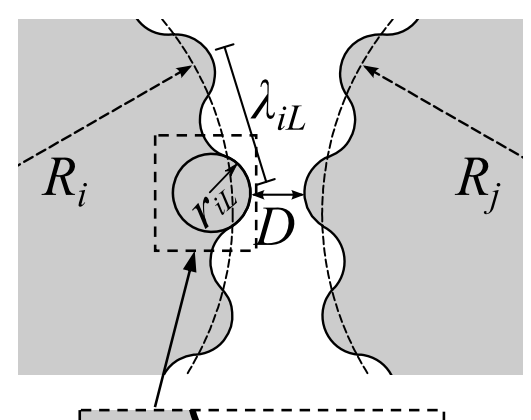

Small scale roughness

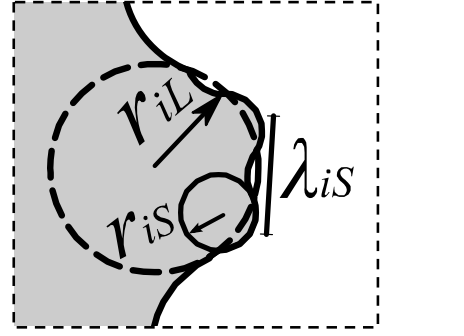

Fig. 2. Schematic drawing of approaching particles with two scales of surface roughness.

The roughness parameters needed in the cohesion model Eq. (10) are listed in Table 1. The average values in Table 1 are used in the base case to study the effect of Young's modules and system scale in section 4.1 and 4.2 , respectively.

Table 1: Roughness parameters used in the cohesion model 


\begin{tabular}{|l|l|l|}
\hline Roughness parameters & Average & Standard deviation \\
\hline$r m s_{L}(\mathrm{~nm})$ & 24 & 8.0 \\
\hline$r m s_{S}(\mathrm{~nm})$ & 2.0 & 1.0 \\
\hline$\lambda_{L}(\mathrm{~nm})$ & 2400 & 400 \\
\hline$\lambda_{S}(\mathrm{~nm})$ & 300 & 100 \\
\hline
\end{tabular}

\subsection{Simulation conditions}

DEM-CFD simulations were performed in a 3D rectangular fluidized bed with a square distributor plate, as shown in Fig. 3. The bed is filled with monodisperse spherical particles with diameter $d_{p}=69 \mu \mathrm{m}$ and density $\rho_{p}=2500 \mathrm{~kg} / \mathrm{m}^{3}$, which is identical to the particles used in experiments. The CFD grid size is 2.5 $d_{p}$, as shown in Fig. 3. Insensitivity of the results was observed as the CFD grid size varied from $1.5 d_{p}$ to $3 d_{p}$. Table 2 summarizes the parameters used in DEM-CFD simulations. The Young's modulus was varied from $10 \mathrm{MPa}$ (artificially soft values) to $73 \mathrm{GPa}$ (actual material value of glass). The static bed height and bed width were controlled by changing the number of particles in simulations from 20,000 to 400,000, corresponding to a variation in static bed height from $0.15 \mathrm{~cm}$ to $1.2 \mathrm{~cm}$ and bed width from $0.14 \mathrm{~cm}$ to $0.48 \mathrm{~cm}$. As our study is to explore the possibility of comparing small-scale simulation with large-scale, there is an intentional mismatch between experimental and DEM-CFD system size. Note that the gas density and viscosity were calculated for ambient air at an altitude of 1,655 m (Bird et al., 2002) since the experiments were conducted in Boulder, Colorado. As the fluidized bed used in experiments is much larger than simulation setup, the effect of side walls must be minimized so that simulation results can be compared directly with experiments. To reduce the side-wall effect, we set side walls to be free-slipping for the gas phase, while frictionless and non-cohesive for the solid phase to mimic a fluidized bed with infinitely large distributor plate, similar to our recent work (LaMarche et al., 2015b). A further increase in bed width does not affect the defluidization curves, which will be discussed in detail in result section. Note simulations with periodic boundary condition can also be used to minimize wall effects, which leads to consistent results with those based on the solid walls incorporating the aforementioned treatments. The particle sliding friction coefficient and normal coefficient of restitution were set to values measured for the glass beads used in experiments. 


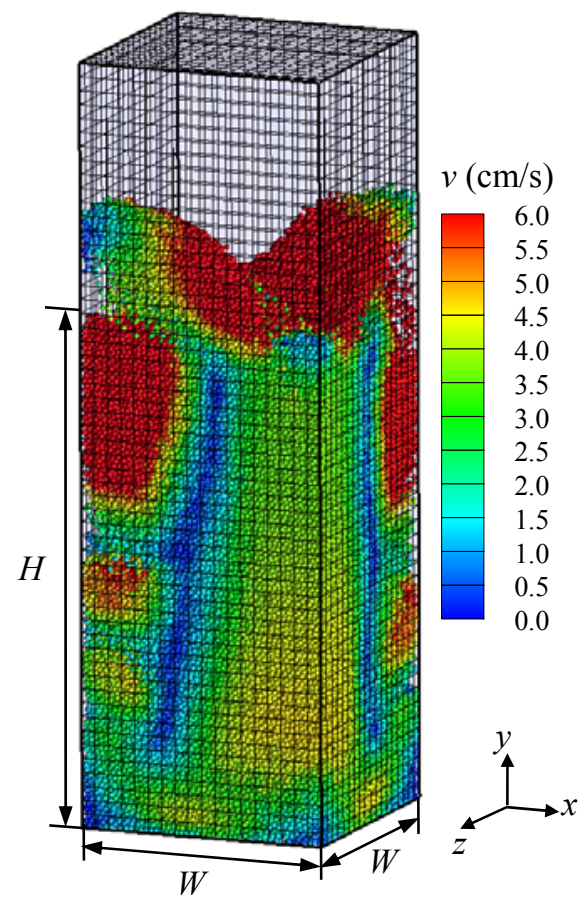

Fig. 3. A snapshot of simulation with $N_{p}=200,000(W=0.32 \mathrm{~cm}, H=0.6 \mathrm{~cm})$ cohesive particles and $E$ $=10 \mathrm{MPa}$ at superficial velocity $U=1.5 \mathrm{~cm} / \mathrm{s}$. The thin lines represent CFD mesh. The color contour indicates particle speed.

Table 2: Parameters used in simulations

\begin{tabular}{|l|l|}
\hline Bed geometry \\
\hline Static bed height, $H(\mathrm{~cm})$ & $0.15-1.20$ \\
\hline Bed width, $W(\mathrm{~cm})$ & $0.14-0.48$ \\
\hline CFD grid size, $\Delta x \times \Delta y \times \Delta z$ & $2.5 d_{p} \times 2.5 d_{p} \times 2.5 d_{p}$ \\
\hline Solid phase & 69 \\
\hline Diameter, $d_{p}(\mu \mathrm{m})$ & 2500 \\
\hline Density, $\rho_{p}\left(\mathrm{~kg} / \mathrm{m}^{3}\right)$ & $2 \times 10^{4}-4 \times 10^{5}$ \\
\hline Total number of particles, $N_{p}$ & $10(\mathrm{softest})-73000$ (glass) \\
\hline Young's modulus, $E(\mathrm{MPa})$ & 0.22 \\
\hline Poisson ratio, $v$ & 0.275 \\
\hline Friction coefficient, $\mu$ & 0.97 \\
\hline Normal coefficient of restitution, $e$ & $3.1 \times 10^{-20}$ \\
\hline Hamaker constant, $A(\mathrm{~J})$ & 0.3 \\
\hline Intermolecular separation, $D_{0}(\mathrm{~nm})$ & 0.95 \\
\hline Particle sphericity, $\psi$ & 0.97 \\
\hline Gas phase & \\
\hline Density, $\rho_{g}\left(\mathrm{~kg} / \mathrm{m}^{3}\right)$ &
\end{tabular}




\begin{tabular}{|l|l|}
\hline Viscosity, $\mu_{g}(\mathrm{~kg} /(\mathrm{m} \cdot \mathrm{s}))$ & $1.8335 \times 10^{-5}$ \\
\hline Superficial velocity, $U(\mathrm{~cm} / \mathrm{s})$ & $0-2.0$ \\
\hline Gas phase time step, $D t(\mathrm{~s})$ & $1.0 \times 10^{-4}$ \\
\hline Courant number, $C$ & $\sim 0.01$ \\
\hline Spatial discretization & Finite Volume \\
\hline Temporal discretization & 1 st order implicit Euler \\
\hline Solver scheme & Superbee \\
\hline
\end{tabular}

The integration time step $d t$ in DEM is given by a fraction of the duration $t$ coll of binary head-on collision between two particles. For non-cohesive impacts, an analytical relation for two identical spheres with radius $R$ is given by (Timoshenko, 1934)

$$
d t=\frac{t_{\text {coll }}}{n}=\frac{4.428}{n}\left(\frac{\rho}{E_{\text {eff }}}\right)^{0.4} \frac{R}{v_{i m}^{0.2}}
$$

where $v_{i m}$ is the impact velocity (relative, normal velocity between particles at time of contact) and $n$ is the number of integration steps during one collision. To determine if cohesion affects this relation, we solved for $t_{\text {coll }}$ numerically for the collision between cohesive particles; the results indicate that collision time is largely unaffected by cohesion. According to previous studies, $n$ should be no less than 5 (van der Hoef et al., 2006), and $n=50$ is considered sufficient (Silbert et al., 2001). To find appropriate values for $n$ and $v_{\text {im }}$ to compute $d t$ for our simulations, we first conducted DEM-CFD simulations using $d t$ based on $n=50$ and $v_{\mathrm{im}}=1 \mathrm{~m} / \mathrm{s}$, assuming that $v_{i m}$ would not exceed $1 \mathrm{~m} / \mathrm{s}$ in our system. By collecting the impact velocities in our simulations, the typical vim was found to be $1 \mathrm{~cm} / \mathrm{s}$. The value of $n$ was then varied from 5 to 50 , and we found insensitivity of simulation results when $n \geq 10$. Therefore, $v_{\mathrm{im}}=1 \mathrm{~cm} / \mathrm{s}$ and $n=10$ were chosen in this work to compute the DEM time step from Eqn. (11). As the time step changes with the Young's modulus in Eq. (11), to ensure the sensitivity of simulation results to Young's modulus to be discussed in section 4.1 is not attributed to the time step difference, we compared the simulation results based on $E=10 \mathrm{MPa}$ using the time step corresponding to $E=10 \mathrm{MPa}$ (softest) and $E=73 \mathrm{MPa}$ (realistic). Identical results were observed between the two simulations.

During the simulations, particles were generated orderly keeping an initial separation distance of $0.05 d_{p}$ so that the interparticle cohesion is negligible before turning on the uniform gas inlet with a superficial velocity of $2 \mathrm{~cm} / \mathrm{s}$, which corresponded to a bubbling regime for all conditions considered in this work. After the simulation reached statistically steady states as revealed by the stabilization of the time 
evolution of particle average velocity, the superficial gas velocity was ramped down to 0 in a stepwise manner with a step size of $0.1 \mathrm{~cm} / \mathrm{s}$. Each gas velocity was held for a duration of $0.1 \mathrm{~s}$ and extension of this duration to $0.3 \mathrm{~s}$ does not affect the results. The bed pressure drop was collected at a sampling frequency of $200 \mathrm{~Hz}$, corresponding to 20 samples per gas velocity. Plotting the averages of sampled pressure drop data at each velocity completes the defluidization curve. Note that the sudden change of gas velocity associated with this stepwise variation occasionally leads to "spikes" of pressure drop, due to transient behavior of particles as discussed by Pandit et al. (2006). To make sure the average value is representative of the steady state, the first 2 points collected for each gas velocity were excluded from the calculation of average pressure drop.

\section{Results and discussion}

\subsection{Effect of Young's modulus}

\subsubsection{Flow patterns}

The effect of Young's modulus was studied with a bed of $N_{p}=50,000$ particles. Fig. 4 shows the flow patterns of cohesive particles at 5 gas superficial velocities under 2 Young's moduli E: $10 \mathrm{MPa}$ represents a typical value used in DEM-CFD and $73 \mathrm{GPa}$ is the material Young's modulus of glass particle. During defluidization, gas velocities decrease and flow pattern evolves from left to right with increasing time. At higher gas velocities $U \geq 1.5 \mathrm{~cm} / \mathrm{s}$, fluidization is in the bubbling regime and motion of particles is vigorous for both Young's moduli, reflected by the relatively high speed of particles. When $U$ decreases to $1.0 \mathrm{~cm} / \mathrm{s}$, particle motion is completely suppressed for $E=10 \mathrm{MPa}$. However, under the material Young's modulus $E=73 \mathrm{GPa}$, a portion of particles with higher velocities remain noticeable possibly as a result of sporadic bubbling which is weak but not totally prevented. With further decreased gas velocity $U \leq 0.5 \mathrm{~cm} / \mathrm{s}$, fluidized beds with $E=10 \mathrm{MPa}$ and $73 \mathrm{GPa}$ both start to behave as a stable system, i.e. non-bubbling, partially fluidized state with much lower particle velocities. The results indicate that higher Young's modulus delayed the occurrence of stable bed to lower gas velocities. This sensitivity to Young's modulus is also reflected in Fig. 5, which shows the corresponding spatial bed porosity at different gas velocities. Consistent with the flow patterns in Fig. 4, the spatial fluctuation of porosity is higher at $U \geq 1.5 \mathrm{~cm} / \mathrm{s}$, indicative of bubbling. As the bed becomes stable at lower gas velocities, the porosity distribution becomes more homogenous. However, packed 
beds at lower gas velocity $U \leq 0.5 \mathrm{~cm} / \mathrm{s}$ are fluffier for $E=10 \mathrm{MPa}$ than $E=73 \mathrm{GPa}$, indicated by the slightly higher overall porosities.

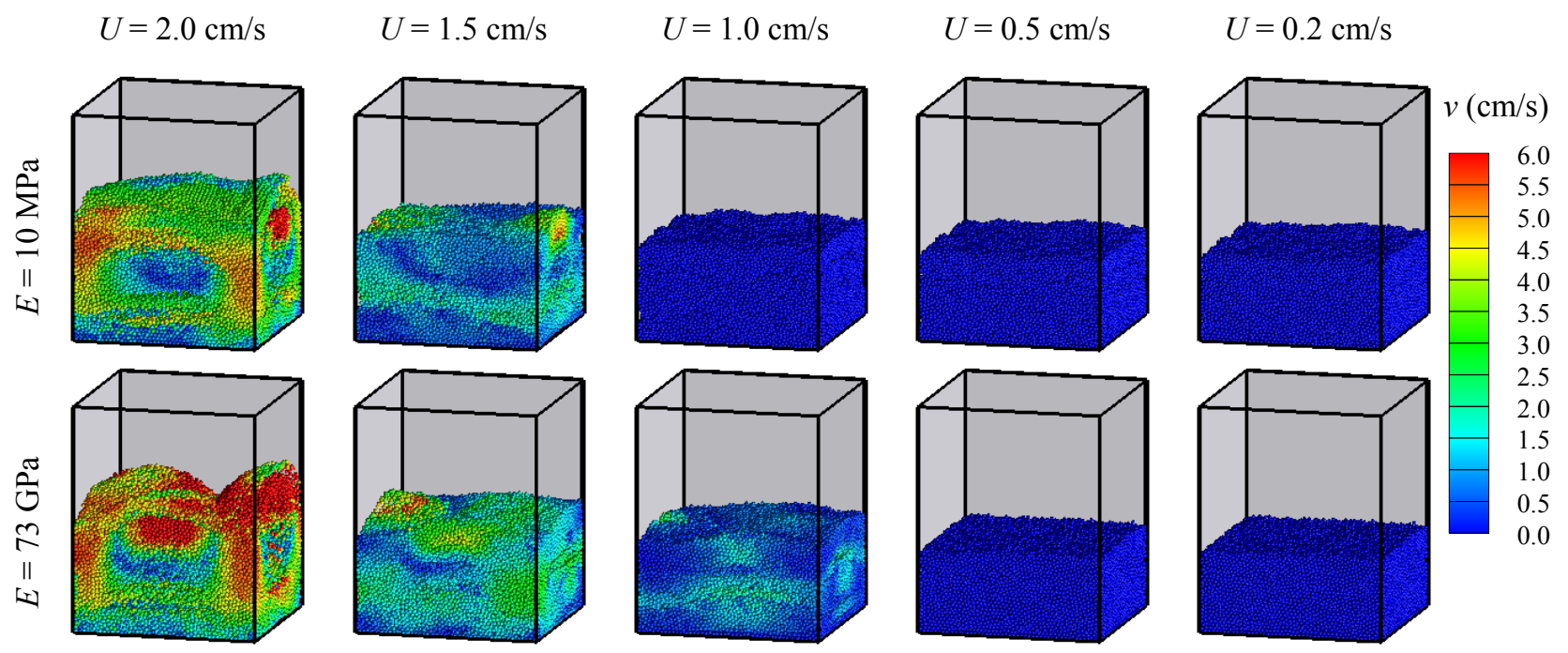

Fig. 4. Flow patterns of cohesive particles at different Young's moduli and superficial gas velocities. $(H$ $\left.=0.15 \mathrm{~cm}, W=0.32 \mathrm{~cm}, N_{p}=50,000\right)$

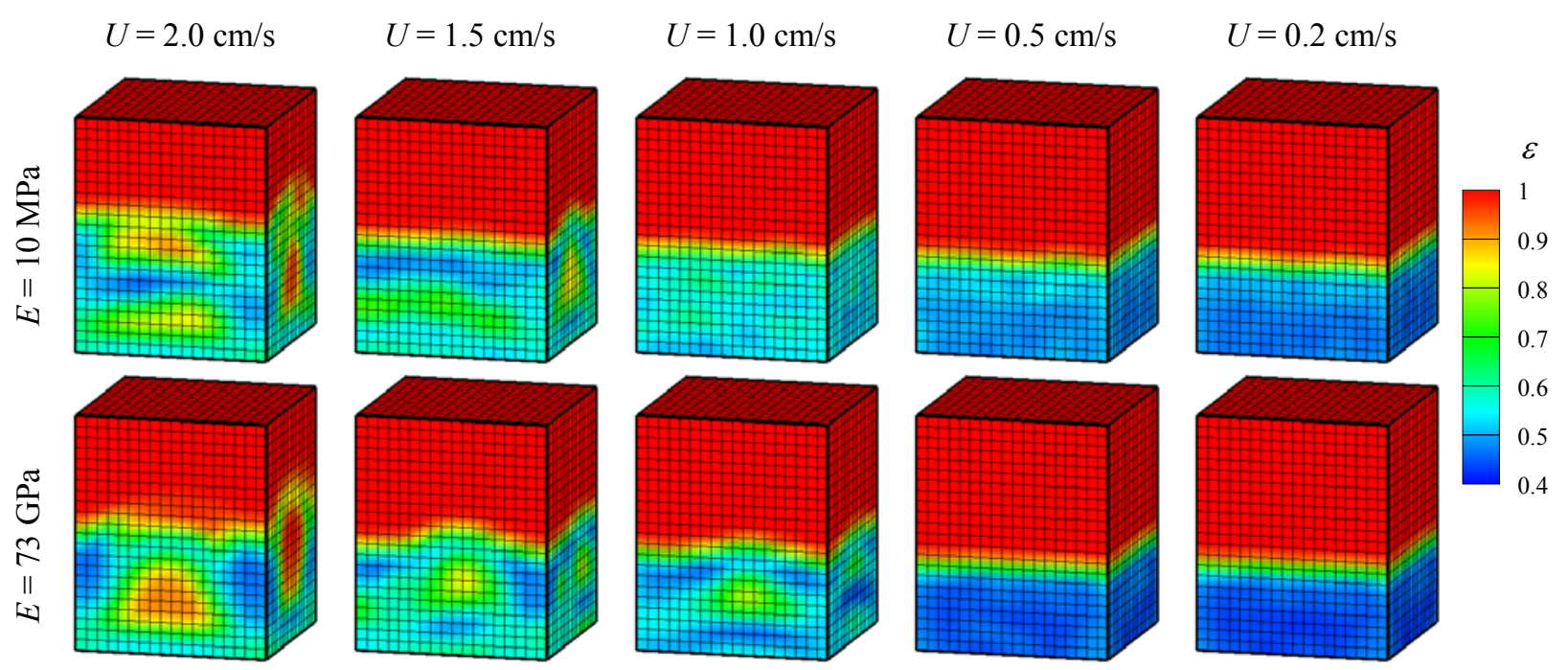

Fig. 5. Porosity of cohesive particles at different Young's moduli and superficial gas velocities. $(H=$

$$
\left.0.15 \mathrm{~cm}, W=0.32 \mathrm{~cm}, N_{\mathrm{p}}=50,000\right)
$$

The sensitivity of Young's modulus can be further revealed by comparing the spatiotemporal evolutions of bed porosity for two Young's moduli in Fig. 6. During bubbling regime under high gas velocity, bed 
porosity along the bed height ( $y$-direction in Fig. 3) has higher fluctuations as indicated by the "bubbling" region in Fig. 6. With decreasing gas velocity, bubbling stops and the bed switches to a stable state, where fluctuation of bed porosity is depressed, as shown by the "stable" region in Fig. 6. Comparing Fig. 6a and b, with increasing Young's modulus from $10 \mathrm{MPa}$ to $73 \mathrm{GPa}$, the critical gas velocity $U_{c r i t}$ at the transition from "bubbling" to "stable" regimes decreases from $1.1 \mathrm{~cm} / \mathrm{s}$ to $0.6 \mathrm{~cm} / \mathrm{s}$.

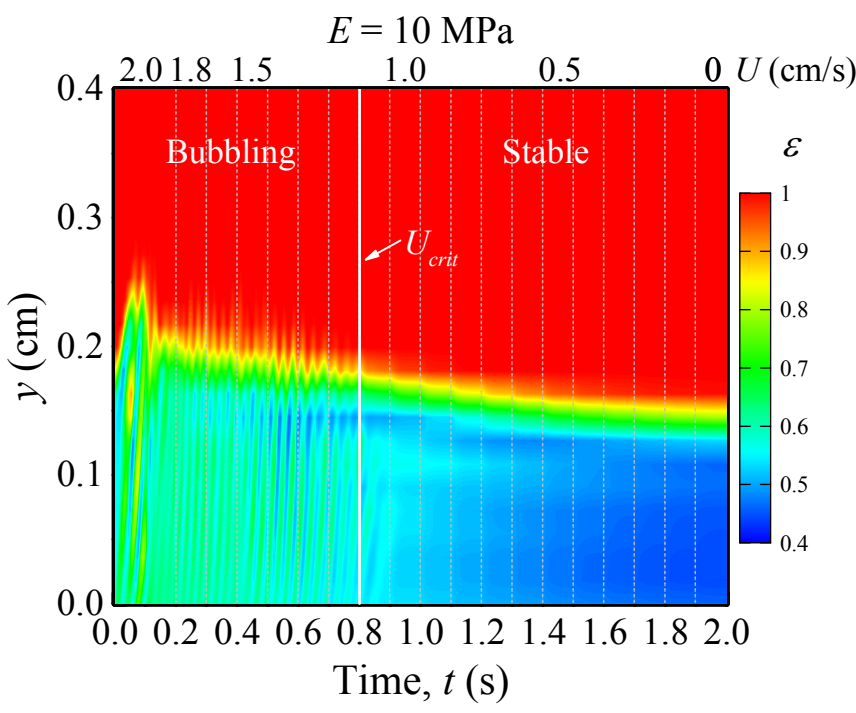

(a)

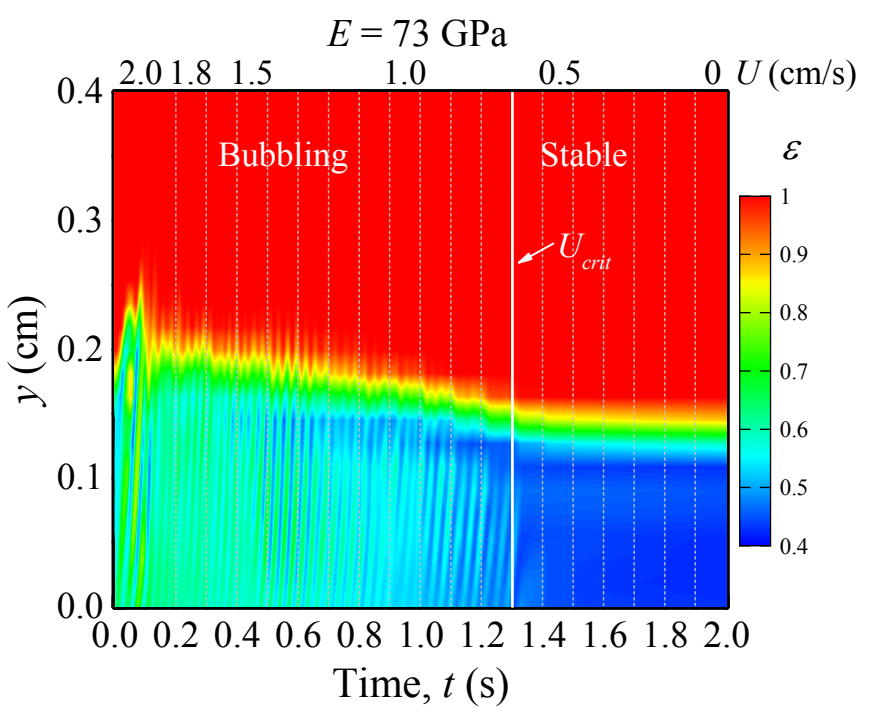

(b)

Fig. 6. Spatiotemporal evolutions of bed porosity profile along the bed height ( $y$-direction in Fig. 3): (a) $E=10 \mathrm{MPa}$ and (b) $E=73 \mathrm{GPa}$. Lower horizontal axis shows the evolution of time, which is separated by dash lines into periods. During each period, a corresponding superficial gas velocity indicated by the upper horizontal axis were held constant. The solid line in each figure separate regions of "bubbling" with high fluctuation of porosity from "stable" with low fluctuation of porosity. The porosity is as an average over the same height and the critical gas velocity $U_{\text {crit }}$ is determined when the standard deviation of bed porosity falls below 0.003 . $\left(H=0.15 \mathrm{~cm}, W=0.32 \mathrm{~cm}, N_{p}=50,000\right)$

\subsubsection{Defluidization curves}

Collectively, the results presented in Figs. 4-6 above indicate that the flow patterns of cohesive particles near the point of defluidization is sensitive to the Young's modulus. Here, we consider the impact on the defluidization curve, which was previously found insensitive to Young's modulus for non-cohesive particles (LaMarche et al., 2015b). Fig. 7 shows the defluidization curves with increasing Young's 
modulus for both non-cohesive and cohesive particles. Similar to previous DEM-CFD results on fluidization (gas velocity increases with time) of particles with or without cohesion (Rhodes et al., 2001; Pandit et al., 2006; Weber and Hrenya, 2007), $\Delta p^{*}$, the pressure drop normalized by particle weight/area of the distributor plate, stays around unity at higher gas velocities, indicating full fluidization. With gas velocity further decreased, $\Delta p^{*}$ decreases monotonically from unity to zero, corresponding to the stable bed with non-fluidization. With increasing Young's modulus, the defluidization curves for cohesive particles changes quite significantly as show in Fig. 7a, where curves are seen converging to the curve with highest $E=73 \mathrm{GPa}$. The gas velocity at the transition from full fluidization $\left(\Delta p^{*} \approx 1\right)$ to nonfluidization $\left(\Delta p^{*}<1\right)$ shifts to smaller values with increasing Young's modulus and becomes relatively insensitive at the two highest Young's moduli. On the other hand, for non-cohesive particles, defluidization curves are insensitive to the varying Young's modulus as all the curves collapse into a single curve, as shown in Fig. $7 \mathrm{~b}$.

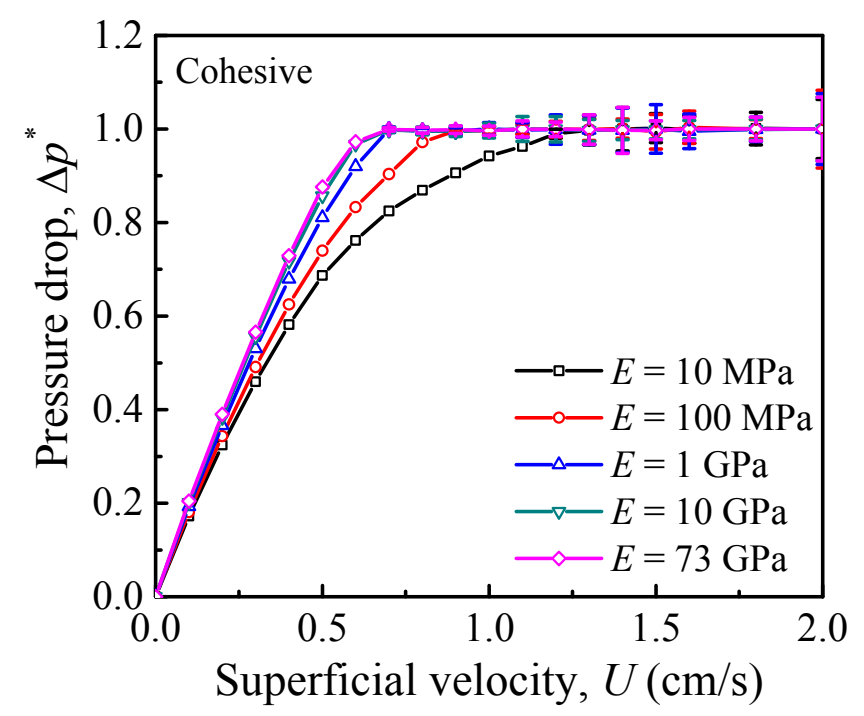

(a)

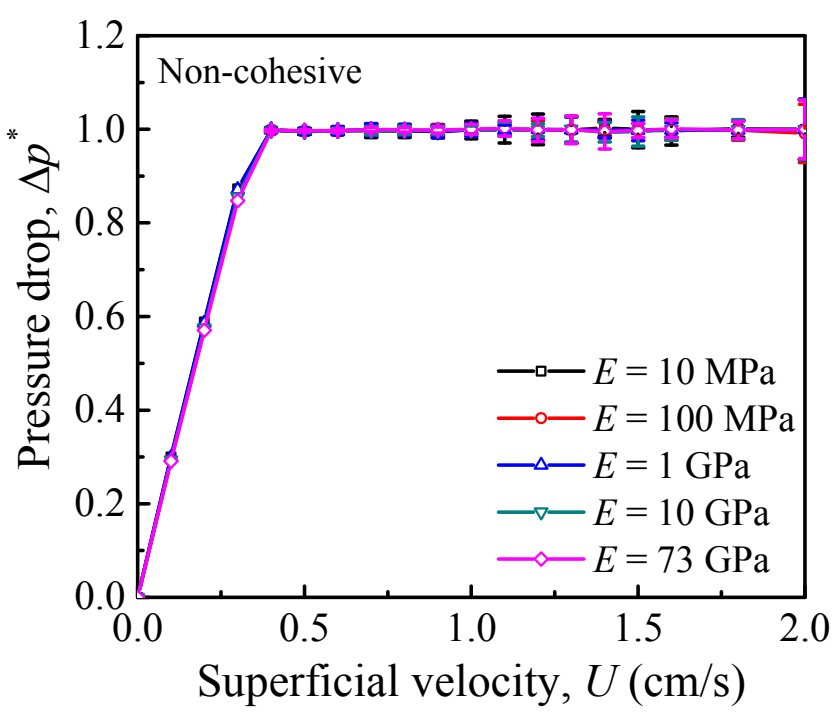

(b)

Fig. 7. Defluidization curves with increasing Young's modulus: (a) cohesive particles and (b) noncohesive particles. $\left(H=0.15 \mathrm{~cm}, W=0.32 \mathrm{~cm}, N_{p}=50,000\right)$. Error bars represent the standard deviation of pressure drop collected at each gas velocity. Note that for cohesive particles, curves with $E=10 \mathrm{GPa}$ and $E=73 \mathrm{GPa}$ almost collapsed and for non-cohesive particles, all curves associated with different Young's modulus are collapsing onto a single curve. 
To quantify the effect of Young's modulus, a characteristic velocity is extracted from the defluidization curves, namely the complete fluidization velocity $U_{c f}$. The method for determining $U_{c f}$ is shown in Fig. 8: $U_{c f}$ is the gas velocity at which the pressure drop falls below $98 \%$ of averaged pressure drop at full fluidization indicated by $\left\langle\Delta p_{F}^{*}\right\rangle$. The $U_{c f}$ as a function of Young's modulus is plotted in Fig. 9. While $U_{c f}$ stays constant for non-cohesive particles, it decreases with increasing Young's modulus and a more dramatic change is seen at smaller Young's moduli.

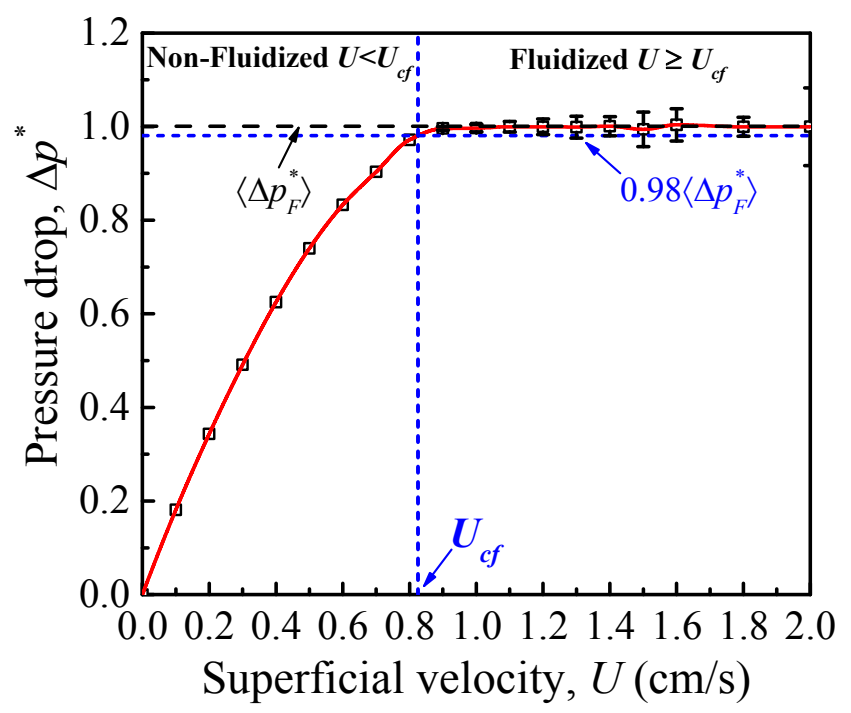

Fig. 8. Illustration of the determination of complete fluidization velocity $\left(U_{c f}\right)$ based on the defluidization curve with $E=100 \mathrm{MPa}, H=0.15 \mathrm{~cm}, W=0.32 \mathrm{~cm}, N_{p}=50,000$.

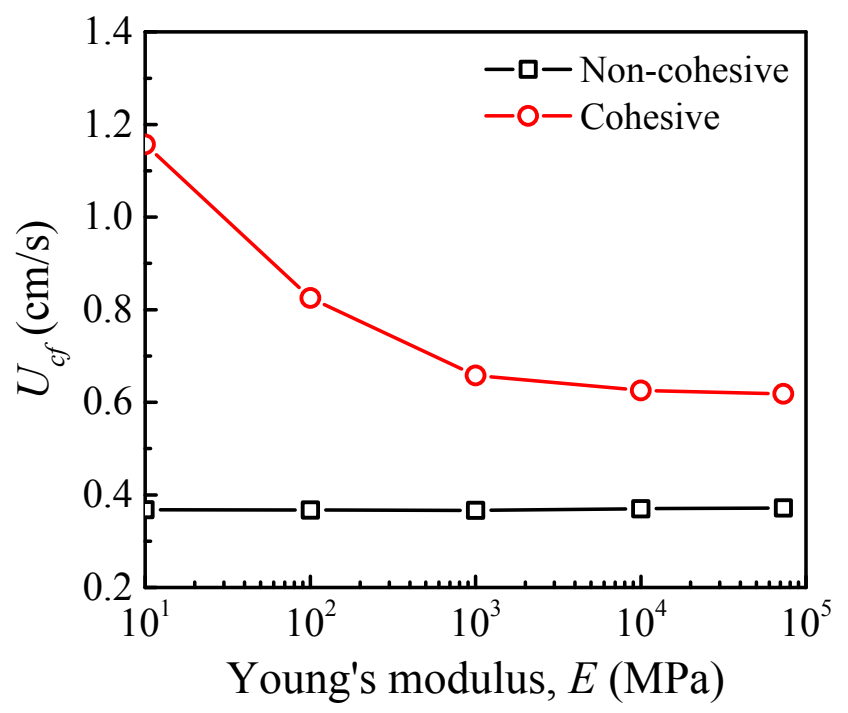


Fig. 9. Variation $U_{c f}$ with increasing Young's modulus. $\left(H=0.15 \mathrm{~cm}, W=0.32 \mathrm{~cm}, N_{p}=50,000\right)$

\subsubsection{Enhanced cohesive effect with lower Young's modulus: a binary collision}

From the observations above, the consequences of using unrealistically small Young's modulus on defluidization include: (i) fluffier bed packing (Fig. 5), (ii) higher bubbling-stable transition gas velocity $U_{c r i t}$ (Fig. 6), and (iii) higher $U_{c f}$ (Fig. 9), all of which can be associated with an increased cohesive effect, as discussed in the following section. This increased cohesive effect at small Young's modulus during defluidization is consistent with the conclusion made based on bubbling bed simulations using a linear contact model (Moreno-Atanasio et al., 2007; Kobayashi et al., 2013).

Their analysis is extended here from particle-wall cohesion based on linear contact model to particleparticle cohesion based on the Hertz contact model. (The latter was used in this study since the Young's modulus in Hertz contact model is a material property with tabulated values available for glass particles. The spring constant required by the linear contact model, however, is not a standard material property.) Through numerical solution of a head-on collision between two cohesive particles, the effective coefficient of restitution $e_{\text {eff }}$, ratio of post-collisional relative velocity to impact velocity $v_{i m}$ (precollisional relative) velocity, is plotted as a function of increasing impact velocity in Fig. 10a. For vim lower than the critical deagglomeration $v_{\text {crit }}$, particles form an agglomerate after collision and $e_{\text {eff }}=0$. With further increased $v_{i m}$, particles separate after collision (bounce) and $e_{\text {eff }}$ increases asymptotically towards the input coefficient of restitution in DEM-CFD; a similar trend of $e_{e f f}$ vs. vim was also reported for wet particles with liquid-bridging cohesion (Gondret et al., 2002; Donahue et al., 2012a; Donahue et al., 2012b; Gollwitzer et al., 2012). The effect of Young's modulus is shown in Fig. 10b, where $v_{\text {crit }}$ increases with decreasing Young's modulus. As a higher $v_{\text {crit }}$ is also associated with a higher cohesive force, using an artificially low Young's modulus effectively increases the cohesive effect. Physically, it can be understood as follows (Moreno-Atanasio et al., 2007): the work needed to separate two particles is the cohesive force integrated over penetration depth during collision. Though the magnitude of cohesion force does not change with Young's modulus, the work associated with different Young's modulus varies - i.e., with smaller Young's modulus (softer particles), the penetration depth duration collision would be larger, leading to increased cohesion work. 


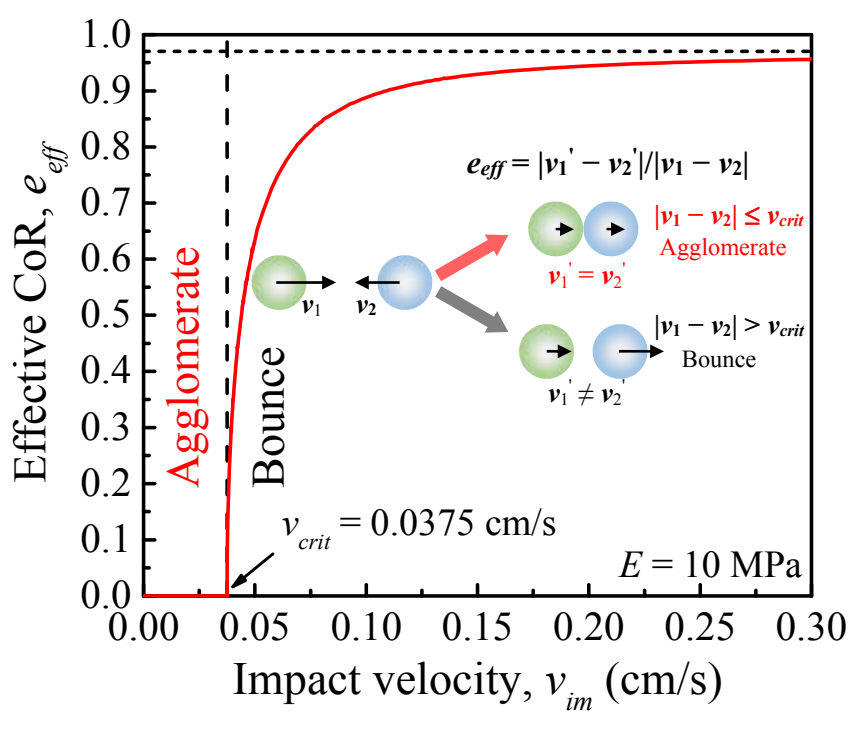

(a)

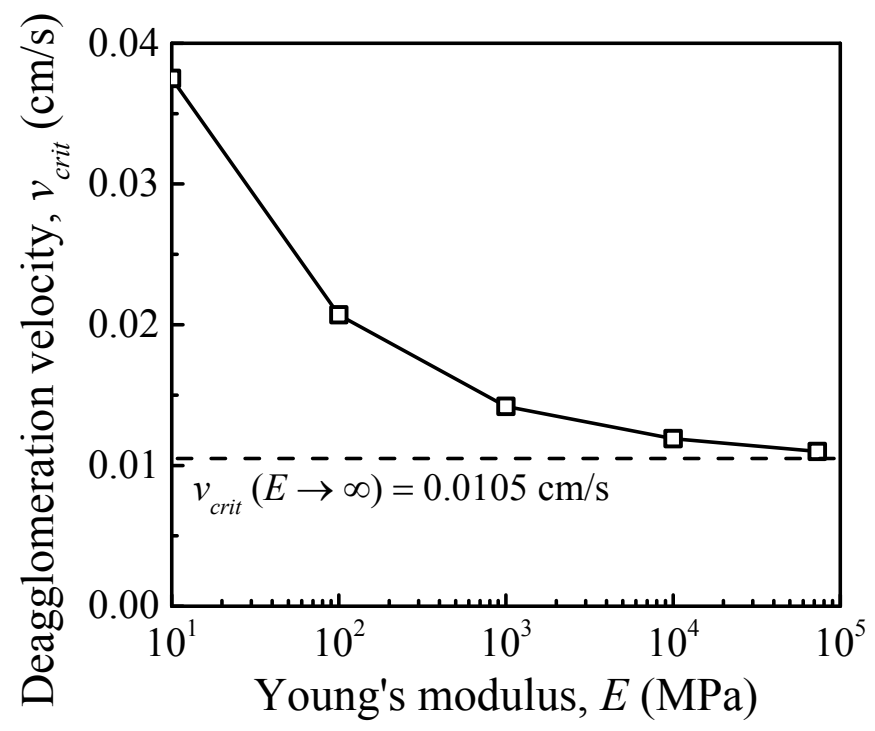

(b)

Fig. 10. (a) Effective coefficient of restitution as a function of impact velocity at $E=10 \mathrm{MPa}$ (b) variation of critical deagglomeration velocity with increasing Young's modulus

\subsubsection{Enhanced cohesive effect with lower Young's modulus: defluidization}

Based on the previous discussion of the enhanced cohesive effect with lower Young's modulus for a binary collision, we now provide further physical insight into how defluidization depends on Young's modulus, as summarized at the beginning of Section 4.1.3. Fig. 11a is a plot of the probability of collisions collected in fluidized beds with impact velocity larger than critical deagglomeration velocity $\left(v_{i m}>v_{c r i t}\right)$, i.e., collisions that will result in "bounce". It is seen that at higher gas velocities where binary collisions are frequent, the probability sharply decreases with decreasing Young's modulus, indicating more particles agglomerate after collisions. With a higher probability of agglomeration, bubble formation will require higher gas velocity to break the agglomerates, leading to a higher bubbling-stable transition gas velocity with decreasing Young's modulus as observed in Fig. 6. Similarly, as a result of increased agglomeration, a decreasing average speed of particles $\langle v\rangle$ at gas velocities corresponding to the bubbling regimes $(\langle v\rangle>0)$ is expected with decreasing Young's modulus, as shown in Fig. 11b. The reduction of average particle velocity at lower Young's modulus may explain the fluffier bed in the stable state, shown in Fig. 5. More specifically, since particles are slowly settling down as gas velocity is decreasing, the defluidization process can be viewed as a packing 
process. An analogy is effect of tapping, during which the particle speed increases, leading to a denser packing of particles in a container after tapping is stopped.

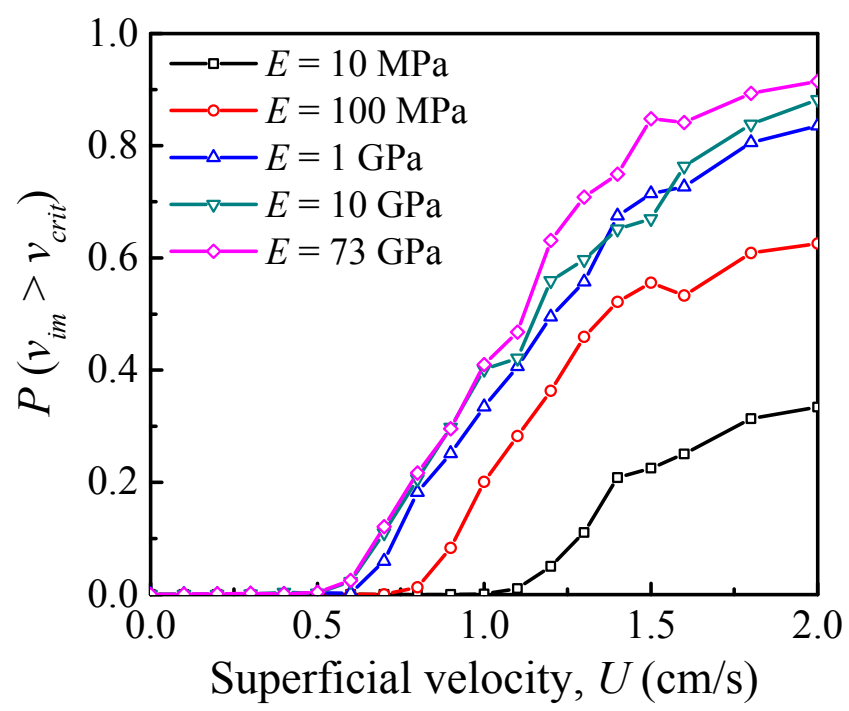

(a)

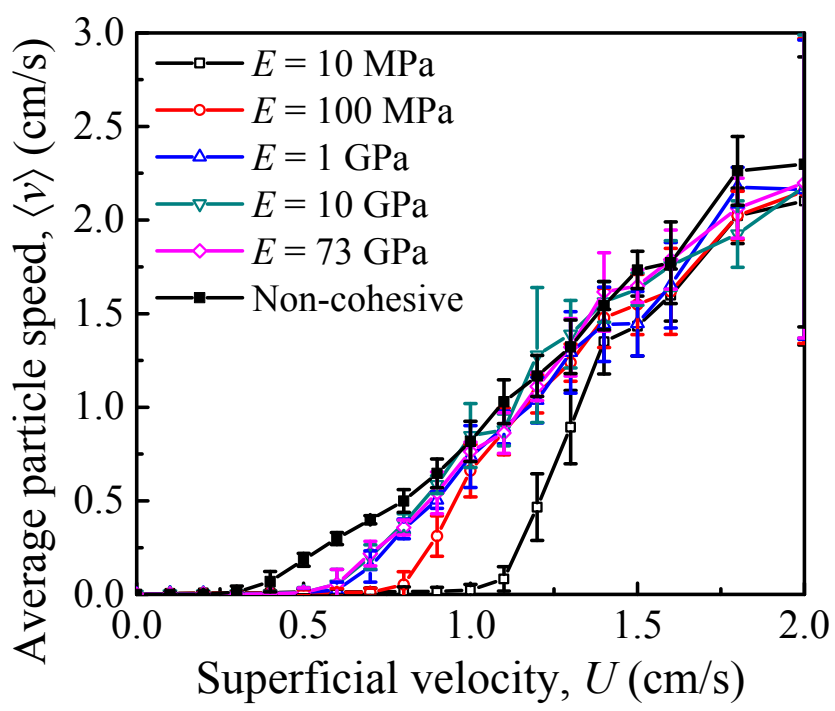

(b)

Fig. 11. (a) Probability of collisions with impact velocity higher than critical deagglomeration velocity and (b) average particle velocity with increasing superficial gas velocity at different Young's modulus. The average velocity for non-cohesive particles is also shown for $E=73 \mathrm{GPa}(H=0.15 \mathrm{~cm}, W=0.32$

$$
\left.\mathrm{cm}, N_{p}=50,000\right)
$$

To quantify the bed fluffiness, the bed porosity profile along the bed height at $U=0.5 \mathrm{~cm} / \mathrm{s}$ and $U=0.2$ $\mathrm{cm} / \mathrm{s}$ is shown in Fig. 12a for cohesive and non-cohesive particles, respectively. Different gas velocities are chosen to ensure the bed has reached the stable state as shown in Fig. 6. For cohesive particles, the porosity decreases with increasing Young's modulus. Since pressure drop through packed bed depends on bed porosity, the variation of bed pressure drop in Fig. 7 at different Young's modulus can be traced to the bed porosity change. In Fig. 12b, we compare the DEM-CFD results and theories of bed pressure drop from Ergun and Carman-Kozeny equations (Rhodes, 2008). The predicted pressure drops were calculated by integrating the profile of bed porosity obtained in DEM-CFD simulations (Fig. 12a) along the bed height $\Delta P=\int_{0}^{H} f[\varepsilon(y)] d y$, where $f$ represents the expression for theoretical equations. Thus, an implicit relation was established between bed pressure drops and Young's moduli. It is shown in Fig. $12 \mathrm{~b}$ that for both theories and DEM-CFD results, the pressure drop increases with Young's modulus, 
which is consistent with the trend shown by defluidization curves in Fig. 7. Thus, the increasing porosity at lower Young's modulus is responsible for the increasing $U_{c f}$ with decreasing Young's modulus in Fig. 9 , i.e., a stable packed bed of higher porosity requires a higher superficial velocity to be fully fluidized due to increased drag. Note that DEM results show better agreement with Carman-Kozeny equation but deviates from the Ergun equation by $\sim 17 \%$. This deviation is probably caused by the underestimated Stokes drag in the Ergun equation for systems with low Reynolds number $\operatorname{Re}=d_{p} U \rho_{g} /\left[\mu_{g}(1-\varepsilon)\right]<1$ (Hill et al., 2001b) and for our system $\operatorname{Re} \approx 0.14$.

For non-cohesive particles, changing Young's modulus does not affect the relative velocities between particles after collision (Antypov and Elliott, 2011), opposite to cohesive particles. Thus, Young's modulus has little effect on the porosity (and correspondingly the bed pressure drop) (Fig. 12b), as well as $U_{c f}$ (Fig. 9). In Fig. 12a, the cohesive particles have higher porosity, which can be ascribed to the "cohesion-enhanced-friction" that allows looser packing to be stable (Yang et al., 2003; Valverde et al., 2004). In particular, since the friction is directly proportional to the normal contact force, as cohesion increases the normal contact force between particles, friction is increased accordingly Weber and Hrenya (2007).
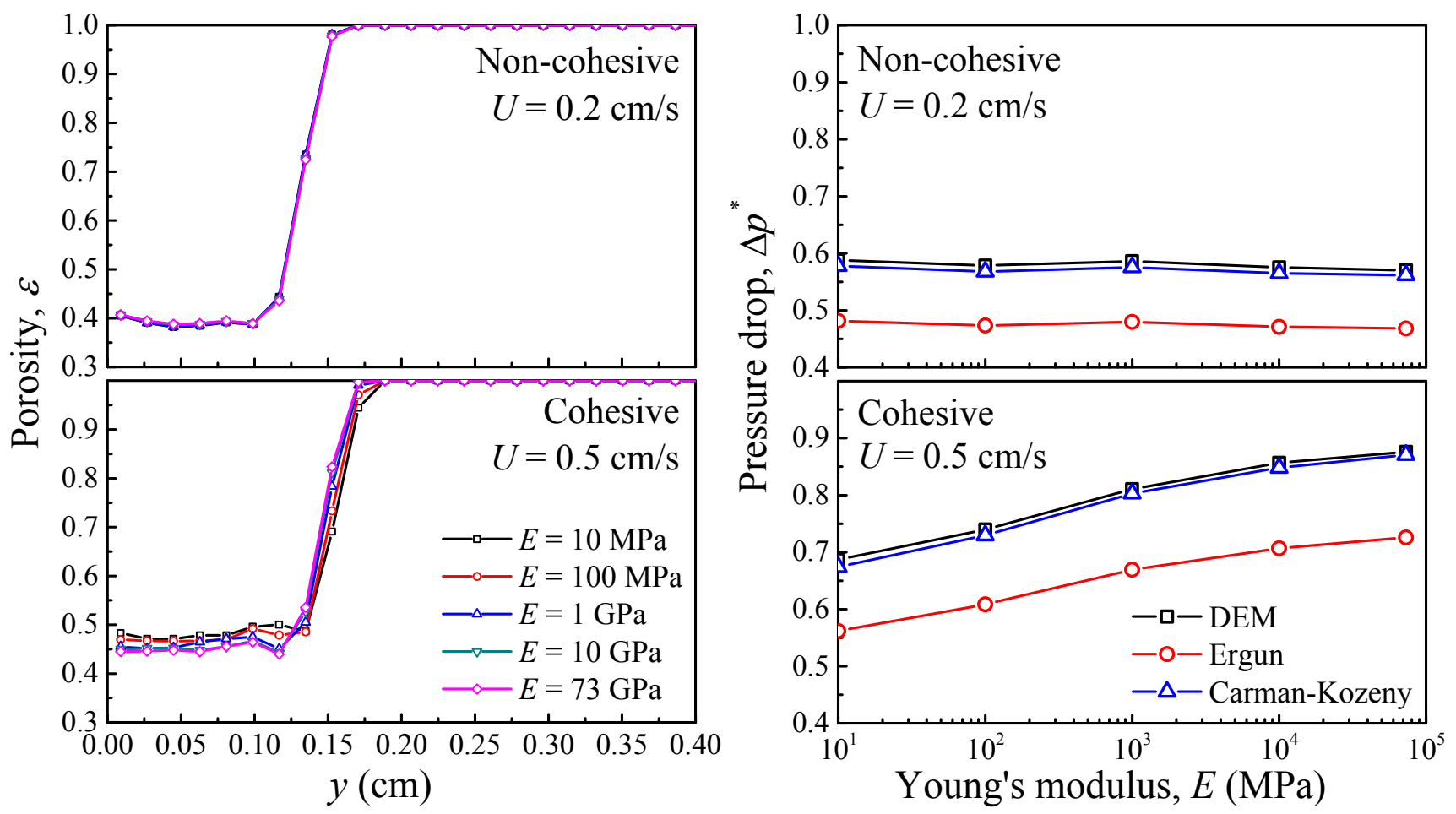
Fig. 12. (a) Bed porosity profile along the direction of bed height ( $y$-direction in Fig. 3) and (b) comparison of the pressure drop between DEM-CFD and theoretical predictions. $(H=0.15 \mathrm{~cm}, W=$ $0.32 \mathrm{~cm}, N_{p}=50,000 ; U=0.5 \mathrm{~cm} / \mathrm{s}$ for cohesive particles, $U=0.2 \mathrm{~cm} / \mathrm{s}$ for non-cohesive particles)

\subsection{Effect of static bed height}

Recall our main goal is to determine if the defluidization curve is independent of system size for cohesive particles, in order to allow for direct comparison between small-scale simulations and largescale experiments. From above results, it is clear that a realistic Young's modulus is needed for such a comparison. Nevertheless, a smaller-than-realistic Young's modulus (10 MPa) is used initially to save simulation time while assessing bed-height dependency, and later the results using realistic Young's modules will be presented for purposes of model validation. To motivate this two-step approach, our simulations using 36 Intel Westmere 2.8Ghz processors of in parallel with $N_{p}=200,000$ take $\sim 4$ days for $E=10 \mathrm{MPa}$ but would take $\sim 84$ days for $E=73 \mathrm{GPa}$ with 36 processors. As shown in Fig. 13 for $E=10 \mathrm{MPa}$, the static bed height was varied from $0.15 \mathrm{~cm}$ to $1.2 \mathrm{~cm}$ by increasing the number of particles in simulations from 50,000 to 400,000 , where the flow transits from static packed bed to bubbling at $U=1.0 \mathrm{~cm} / \mathrm{s}$. Defluidization curves for both non-cohesive and cohesive particles are shown in Fig. 14. For cohesive particles, curves with $\Delta p^{*}<1$ shift to the left, as shown in Fig. 14a. However, for non-cohesive particles, Fig. 14b shows that the defluidization curves have much less dependence of bed height, similar to previous experimental findings (Escudero and Heindel, 2011; Sanchez-Delgado et al., 2011). Correspondingly, the $U_{c f}$ determined from defluidization curves is decreasing with increasing static bed height for cohesive particles, and largely unaffected for non-cohesive particles, as shown in Fig. 15. 


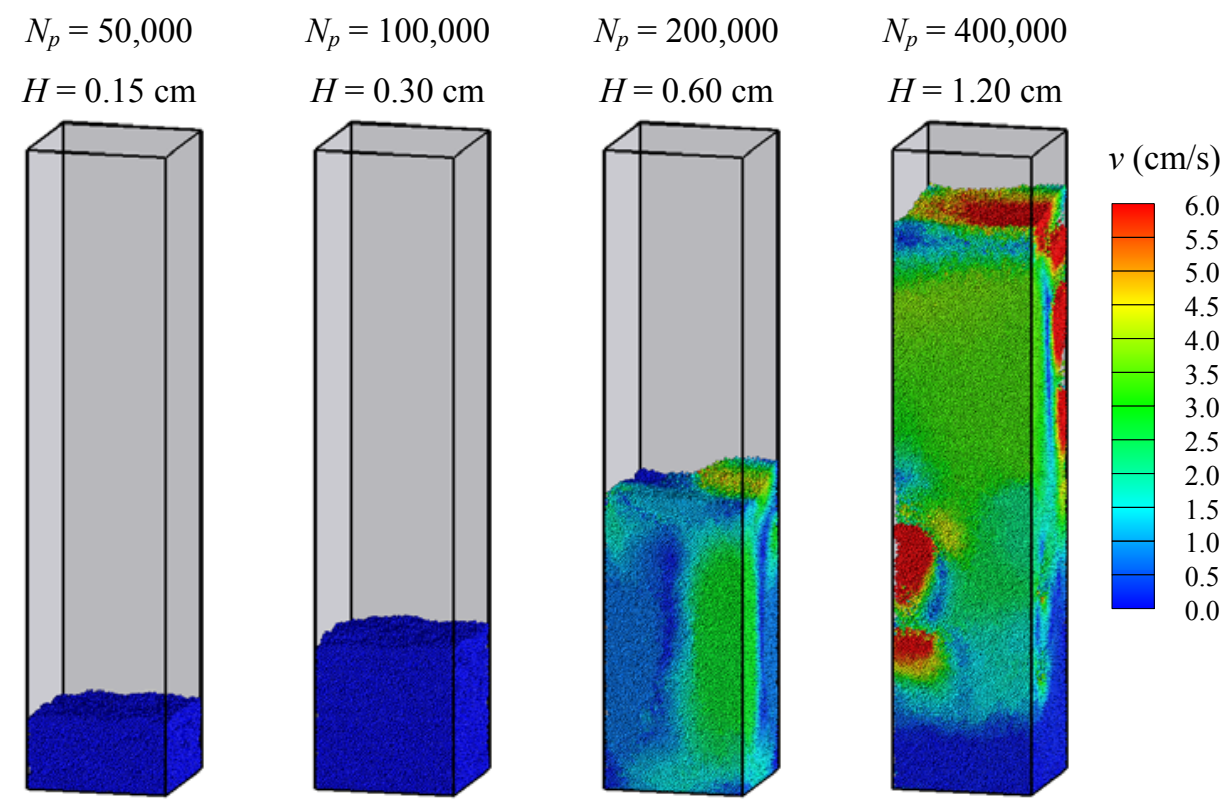

Fig. 13. Flow patterns of cohesive particles at different bed height at superficial gas velocity $U=1.0$ $\mathrm{cm} / \mathrm{s}(W=0.32 \mathrm{~cm}, E=10 \mathrm{MPa})$

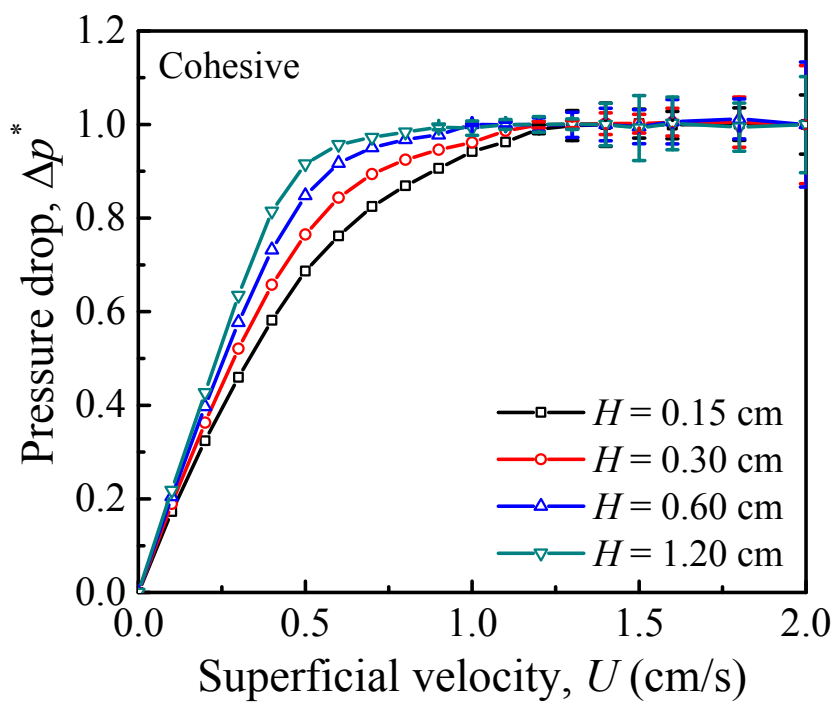

(a)

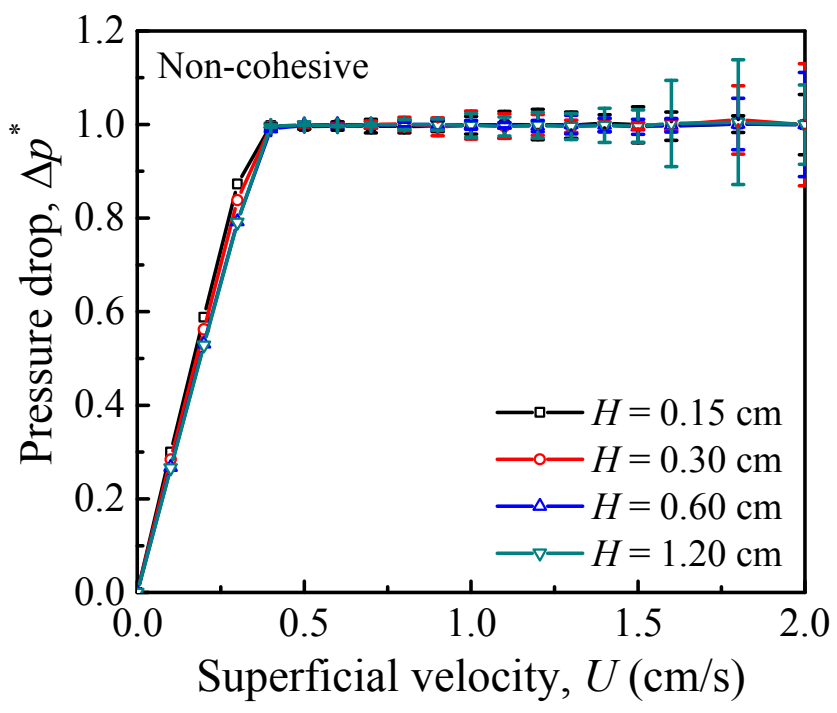

(b)

Fig. 14. Defluidization curves with varying bed height: (a) cohesive particles and (b) non-cohesive particles. $(W=0.32 \mathrm{~cm}, E=10 \mathrm{MPa})$ 


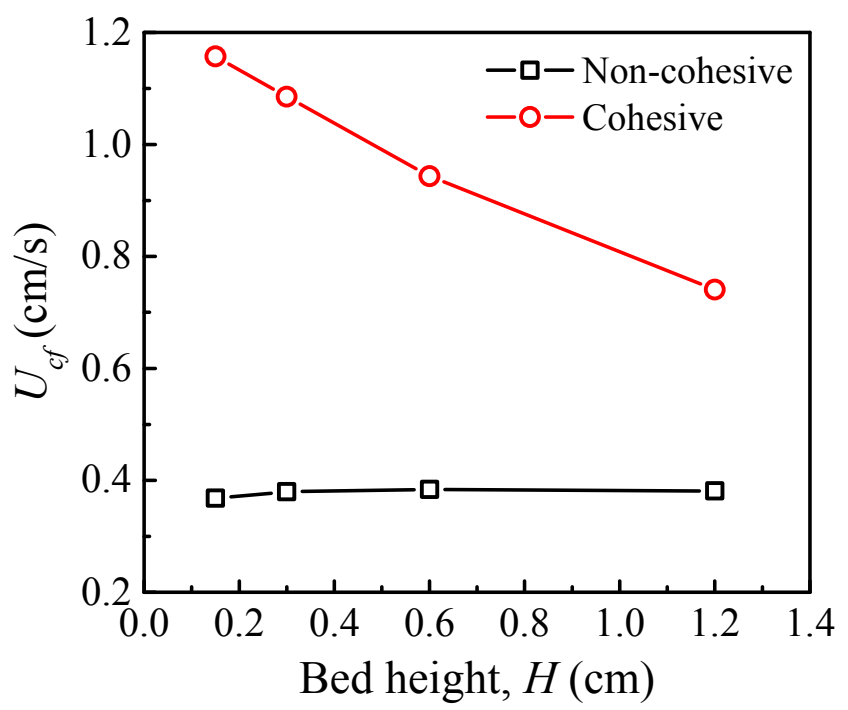

Fig. 15. Variation of $U_{c f}$ with increasing bed height. $(W=0.32 \mathrm{~cm}, E=10 \mathrm{MPa})$

This effect of static bed height can be traced to the variation of bed porosities in the stable regime. As shown in Fig. 16, for cohesive particles, bed porosity decreases with increasing static bed height. Similar to the explanation for the effect of Young's modulus, the decreasing bed porosity with increasing bed height leads to an increase of bed pressure drop at a fixed superficial gas velocity, thereby resulting in the leftward shift of defluidization curves and a decrease in $U_{c f}$, as bed height increases (Fig. 14a). For non-cohesive particles, the bed porosity profiles expose less variation and converges at the highest bed height (Fig. 16) as well as the bed pressure drop, and thus little change of defluidization curves with increasing bed height is observed in Fig. 14b.

For cohesive particles, the decreased porosity with increasing bed height can be understood by the increased solid-phase compressive stress with increasing static bed height, consistent with previous findings (Makse et al., 2000; Yang et al., 2003; Valverde and Castellanos, 2006). During nonfluidization $\left(\Delta p^{*}<1\right)$, when the bed is no longer fully supported by the gas-solid drag, particles at a given height above the distributor will experience more pressure from particles above for a taller bed height. This increased pressure will compress particles to form a denser bed with lower porosity. For non-cohesive particles, though particles also feel higher stress for higher beds, they already pack tightly with porosity fluctuating mildly around the random loose packing with $\varepsilon \approx 0.4$ (Fig. 12a and Fig. 16) (Schroter et al., 2005). As a result, the bed porosity will have less space for variation than cohesive 
particles with increasing compression, causing the relative insensitivity in bed pressure drop and defluidization curves with varying static bed height.

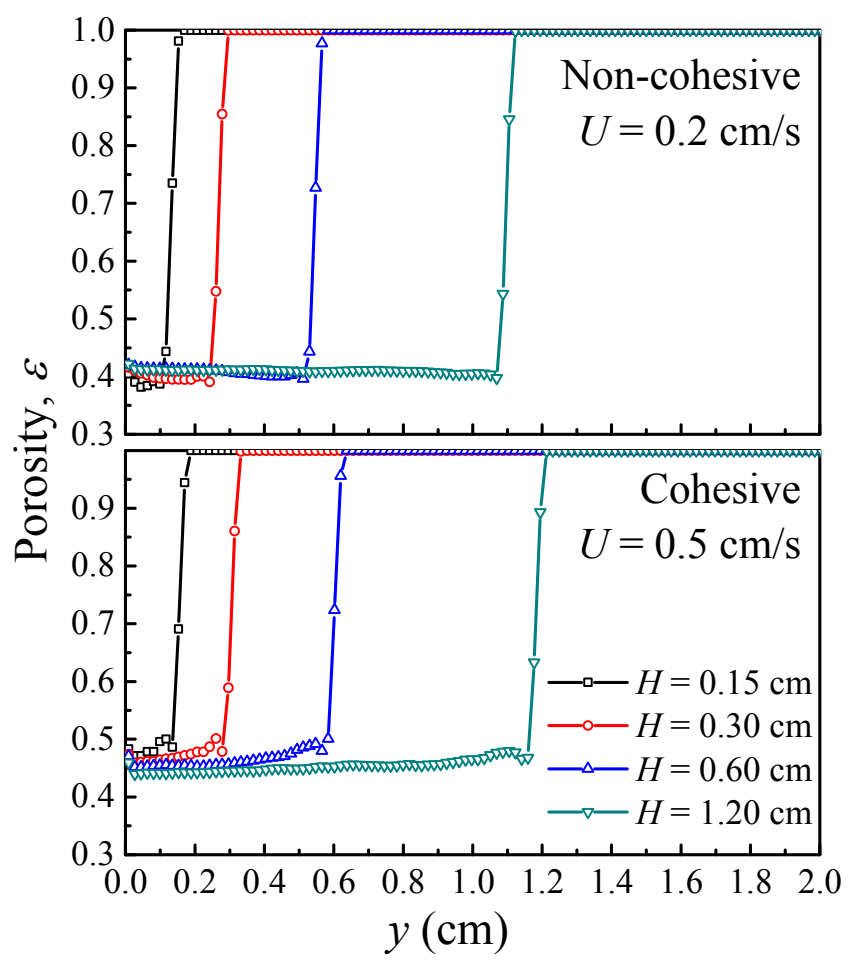

Fig. 16. Bed porosity profile along the direction of bed height ( $y$-direction in Fig. 3). ( $W=0.32 \mathrm{~cm}, E=$ $10 \mathrm{MPa})$

Simulations were also conducted by varying the bed width $W$ from $0.14-0.48 \mathrm{~cm}$ while keeping a constant the static bed height of $0.3 \mathrm{~cm}$ for cohesive particles. Little dependence of defluidization curves as well as the bed porosity profile at stable regime on bed width is found (results not shown). This observation provides further evidence for our hypothesis that it is the changing of compressive stress in the vertical direction that is driving the variation of bed porosity.

\subsection{Comparison with experiments}

Because both the Young's modulus and static bed height affect the cohesive defluidization, care must be taken with both of these parameters in order to make meaningful comparison with experiments. First, the material Young's modulus must be used in DEM-CFD, rather than an artificially soft value. It is worth mentioning that using a previous scaling law that rescales the magnitude of cohesion for 
artificially soft collisions (lower spring constants) such that the post-collisional velocity for a binary collision is kept constant (Kobayashi et al., 2013) is not applicable in our system, even though a similar scaling law can be derived for the Hertz model. The reason is twofold: (i) the scaling law is derived assuming a constant cohesion during contact only, which is not the case for our cohesion model which include a non-contact (short-range) attraction, and more importantly, (ii) the scaling law is developed for binary collisions, which is the dominant particle interaction in bubbling regime (Kobayashi et al., 2013). In defluidization, however, lasting contacts, particle agglomeration and frictional effects are taking dominance over binary collisions.

Second, a sufficient static bed height must be used in DEM-CFD such that the experimental variable of interest (e.g. $U_{c f}$ ) is insensitive to further increases in bed height. Achieving the bed height independence is essential if comparison is conducted between small-scale DEM-CFD simulations and a large experiment, as highlighted in ref. (LaMarche et al., 2015b). For this work, the simulations were conducted with the materials Young's modulus of $73 \mathrm{GPa}$ and increasing the bed height under a constant bed width of $0.32 \mathrm{~cm}$. The results are shown in Fig. 17, where $U_{c f}$ decreases with increasing bed height. The relative change of $U_{c f}$, however, decreases below $1 \%$ for bed height ranging from $1.2 \mathrm{~cm}$ to $1.8 \mathrm{~cm}$. The converging of $U_{c f}$ with increasing bed height here as opposed to the linear decrease of $U_{c f}$ observed in Fig. 15 is likely a result of the reduced cohesive effect at increased Young's modulus, so that $U_{c f}$ is behaving closer to the non-cohesive limit with vanishing sensitivity to bed height. Given the computational restrictions for simulating even higher beds, we consider a bed height of $1.2-1.8 \mathrm{~cm}$ is reasonable for comparison with experiments. Fig. 18 shows such a comparison between experiments and DEM-CFD simulations. As a contrast, different combinations of Young's modulus and bed heights are also shown. Better agreement is seen using the realistic Young's modulus and the highest bed height. Given the uncertainties in the particle roughness measurements, which are illustrated via error bars in the simulation results, the comparison between the experiments and simulations is good when the realistic Young's modulus and sufficient bed height are used in DEM-CFD simulations. 


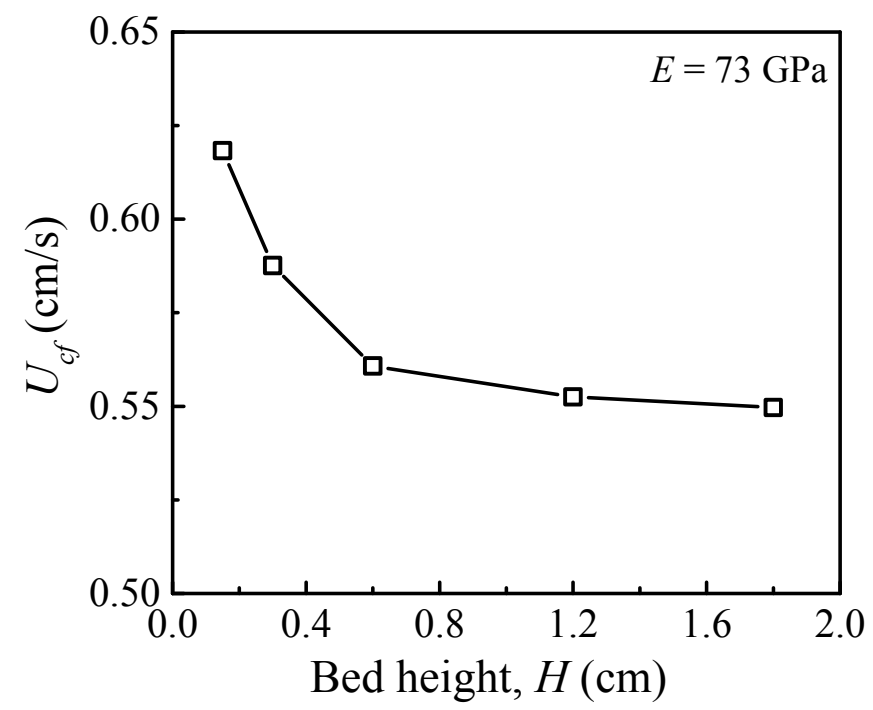

Fig. 17. Variation of $U_{c f}$ with increasing bed height $(W=0.32 \mathrm{~cm}, E=73 \mathrm{GPa})$

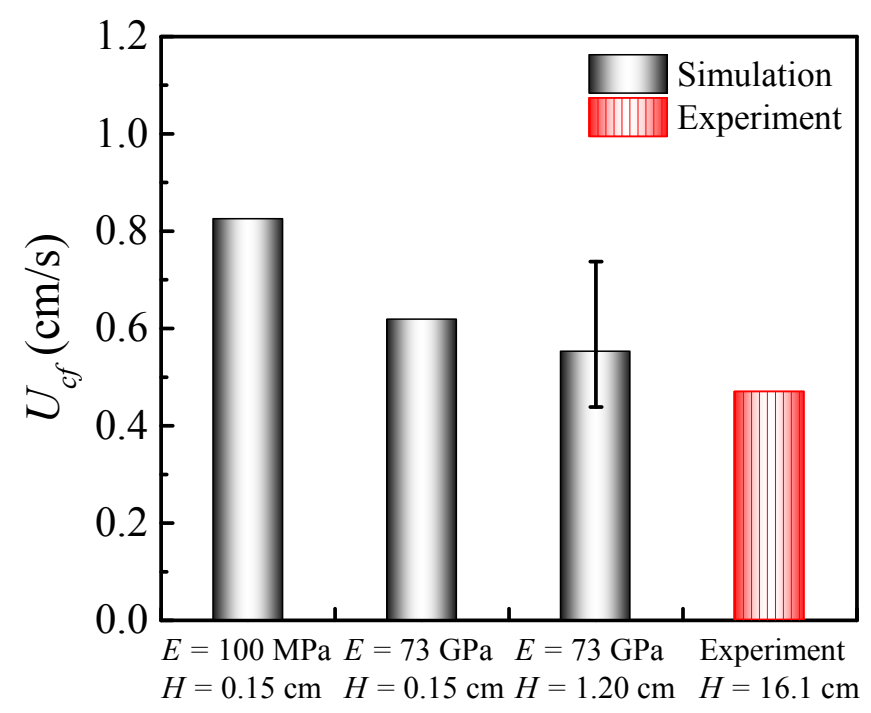

Fig. 18. Comparison of $U_{c f}$ between experiments and DEM-CFD with different Young's modulus and bed height (The error bar on the third column is related to the uncertainties associated with roughness parameters in the cohesion model, as shown by the standard deviations in Table 1).

It is worth noting that the absolute decrease of $U_{c f}$ with increasing Young's modulus from $10 \mathrm{MPa}$ to 73 GPa reduced from $0.54 \mathrm{~cm} / \mathrm{s}$ to $0.19 \mathrm{~cm} / \mathrm{s}$ as bed height increases from $0.15 \mathrm{~cm}$ to $1.2 \mathrm{~cm}$. Thus the sensitivity of $U_{c f}$ to Young's modulus seems to decay with increasing bed height, making it plausible to use unrealistically small Young's modulus in simulations provided the bed height is large. However, 
since the critical height above which the sensitivity to Young's modulus vanishes is unknown a priori (this critical height is possibly dependent on cohesion level and other material properties), we caution that a sensitivity test of the Young's modulus up to the true values is still a crucial prerequisite for conducting systematic DEM-CFD simulations for cohesive particles. Admittedly, due to the short time step required for the true Young's modulus, the simulation in this work with the largest bed height takes approximately one month with 168 processors running in parallel. Practical systems calling for simulations of even larger scale may be infeasible if a quick turnover time is preferred. Therefore, pursing scale-independence within an acceptable time frame serves as an additional motivation for enhancing the efficiency of the DEM-CFD solvers, an on-going effort among many research groups.

\section{Conclusions}

The defluidization of fine cohesive particles was studied via DEM-CFD simulations and experiments, in search of a system-size independent validation measurement and inspired by our previous work for noncohesive particles (LaMarche et al., 2015b). Unlike non-cohesive particles, simulation results for cohesive particles are found sensitive to Young's modulus and static bed height, which stems from the impact of these quantities on cohesion and packing of particles. To make the model validation via comparison between small-scale DEM-CFD simulations and large-scale experiments meaningful, (i) simulating the (non-softened) material Young's modulus and (ii) using a sufficient static bed height at which scale-independence is achieved, are necessary. By meeting both requirements, quantitative agreement between DEM-CFD simulations and experiments in terms of complete fluidization velocity $\left(U_{c f}\right)$ is reached given the uncertainty in cohesive force, in contrast with simulations based on artificially soft particles and insufficient bed height. $U_{c f}$ is therefore identified as a candidate for a system-sizeindependent measurement, which makes simulations based on small-scale systems eligible for direct comparison with large-scale experiments. Such comparison is an essential step in model validation, serving as a foundation for application of DEM-CFD in understanding gas-solid flows.

The results of this work also highlight the caution needed when interpreting DEM-CFD results

obtained based on unrealistically small Young's modulus and small-scale systems, particular for fine particles where cohesive effect is significant. 


\section{Acknowledgements}

The authors are grateful for the financial support provided by the Dow Corning Corporation. This work utilized the Janus supercomputer, which is supported by the National Science Foundation (award number CNS-0821794) and the University of Colorado Boulder. The Janus supercomputer is a joint effort of the University of Colorado Boulder, the University of Colorado Denver and the National Center for Atmospheric Research. Janus is operated by the University of Colorado Boulder.

\section{References}

Antypov, D., Elliott, J.A., 2011. On an analytical solution for the damped Hertzian spring. Europhys. Lett. 94.

Benyahia, S., Syamlal, M., O'Brien, T.J., 2006. Extension of Hill-Koch-Ladd drag correlation over all ranges of Reynolds number and solids volume fraction. Powder Technol. 162, 166-174.

Bird, R.B., Stewart, W.E., Lightfoot, E.N., 2002. Transport phenomena, 2nd ed. J. Wiley, New York.

Castellanos, A., 2005. The relationship between attractive interparticle forces and bulk behaviour in dry and uncharged fine powders. Adv. Phys. 54, 263-376.

Castellanos, A., Valverde, J.M., Perez, A.T., Ramos, A., Watson, P.K., 1999. Flow regimes in fine cohesive powders. Phys. Rev. Lett. 82, 1156-1159.

Chew, J.W., Wolz, J.R., Hrenya, C.M., 2010. Axial Segregation in Bubbling Gas-Fluidized Beds with Gaussian and Lognormal Distributions of Geldart Group B Particles. AIChE J. 56, 3049-3061.

Di Maio, F.P., Di Renzo, A., 2004. Analytical solution for the problem of frictional-elastic collisions of spherical particles using the linear model. Chem. Eng. Sci. 59, 3461-3475.

Ding, J., Gidaspow, D., 1994. A semi-empirical model for fluidization of fine particles. Indian Chem Engr. Section A. 36, 139-150.

Donahue, C.M., Brewer, W.M., Davis, R.H., Hrenya, C.M., 2012a. Agglomeration and deagglomeration of rotating wet doublets. J. Fluid Mech. 708, 128-148.

Donahue, C.M., Davis, R.H., Kantak, A.A., Hrenya, C.M., 2012b. Mechanisms for agglomeration and deagglomeration following oblique collisions of wet particles. Phys. Rev. E 86.

Escudero, D., Heindel, T.J., 2011. Bed height and material density effects on fluidized bed hydrodynamics. Chem. Eng. Sci. 66, 3648-3655. 
Fan, R., Marchisio, D.L., Fox, R.O., 2004. Application of the direct quadrature method of moments to polydisperse gas-solid fluidized beds. Powder Technol. 139, 7-20.

Foerster, S.F., Louge, M.Y., Chang, A.H., Allia, K., 1994. Measurements of the collision properties of small spheres. Phys. Fluids 6, 1108-1115.

Galvin, J.E., Benyahia, S., 2014. The Effect of Cohesive Forces on the Fluidization of Aeratable Powders. AIChE J. 60, 473-484.

Garg, R., Galvin, J., Li, T.W., Pannala, S., 2012. Open-source MFIX-DEM software for gas-solids flows: Part I-Verification studies. Powder Technol. 220, 122-137.

Geldart, D., 1973. Types of gas fluidization. Powder Technol. 7, 285-292.

Geromichalos, D., Kohonen, M.M., Mugele, F., Herminghaus, S., 2003. Mixing and condensation in a wet granular medium. Phys. Rev. Lett. 90, 168702.

Goldschmidt, M.J.V., Kuipers, J.A.M., van Swaaij, W.P.M., 2001. Hydrodynamic modelling of dense gas-fluidised beds using the kinetic theory of granular flow: effect of coefficient of restitution on bed dynamics. Chem. Eng. Sci. 56, 571-578.

Gollwitzer, F., Rehberg, I., Kruelle, C.A., Huang, K., 2012. Coefficient of restitution for wet particles. Phys. Rev. E 86.

Gondret, P., Lance, M., Petit, L., 2002. Bouncing motion of spherical particles in fluids. Phys. Fluids 14, 643-652.

Guo, Y., Curtis, J.S., 2015. Discrete Element Method Simulations for Complex Granular Flows. Annu. Rev. Fluid Mech. 47, 21-46.

Halsey, T.C., Levine, A.J., 1998. How sandcastles fall. Phys. Rev. Lett. 80, 3141-3144.

Hamaker, H.C., 1937. The London - Van Der Waals attraction between spherical particles. Physica 4, 1058-1072.

Hill, R.J., Koch, D.L., Ladd, A.J.C., 2001a. The first effects of fluid inertia on flows in ordered and random arrays of spheres. J. Fluid Mech. 448, 213-241.

Hill, R.J., Koch, D.L., Ladd, A.J.C., 2001b. Moderate-Reynolds-number flows in ordered and random arrays of spheres. J. Fluid Mech. 448, 243-278.

Hou, Q.F., Zhou, Z.Y., Yu, A.B., 2012. Micromechanical modeling and analysis of different flow regimes in gas fluidization. Chem. Eng. Sci. 84, 449-468.

Israelachvili, J.N., 2011. Intermolecular and surface forces, 3rd ed. Academic Press, Burlington, Mass. 
Johnson, K.L., 1985. Contact mechanics. Cambridge University Press, Cambridge Cambridgeshire ; New York.

Kim, H., Arastoopour, H., 2002. Extension of kinetic theory to cohesive particle flow. Powder Technol. $122,83-94$.

Kobayashi, T., Tanaka, T., Shimada, N., Kawaguchi, T., 2013. DEM-CFD analysis of fluidization behavior of Geldart Group A particles using a dynamic adhesion force model. Powder Technol. 248, 143-152.

Kunii, D., Levenspiel, O., 1991. Fluidization engineering, 2nd ed. Butterworth-Heinemann, Boston.

LaMarche, C.Q., Leadley, S., Liu, P., Kellogg, K.M., Hrenya, C.M., 2015a. Method of quantifying surface roughness for accurate adhesive force predictions (submitted).

LaMarche, C.Q., Liu, P.Y., Kellogg, K.M., Weimer, A.W., Hrenya, C.M., 2015b. A system-size independent validation of CFD-DEM for noncohesive particles. AIChE J. 61, 4051-4058.

LaMarche, C.Q., Miller, A.W., Liu, P.Y., Hrenya, C.M., 2015c. Linking micro-scale predictions of capillary forces to macro-scale fluidization experiments in humid environments (submitted).

Li, H.M., McCarthy, J.J., 2003. Controlling cohesive particle mixing and segregation. Phys. Rev. Lett. 90,184301 .

Liu, P.Y., Hrenya, C.M., 2014. Challenges of DEM: I. Competing bottlenecks in parallelization of gassolid flows. Powder Technol. 264, 620-626.

Makse, H.A., Johnson, D.L., Schwartz, L.M., 2000. Packing of compressible granular materials. Phys. Rev. Lett. 84, 4160-4163.

Massimilla, L., Donsi, G., 1976. Cohesive forces between particles of fluid-bed catalysts. Powder Technol. 15, 253-260.

Mikami, T., Kamiya, H., Horio, M., 1998. Numerical simulation of cohesive powder behavior in a fluidized bed. Chem. Eng. Sci. 53, 1927-1940.

Moreno-Atanasio, R., Xu, B.H., Ghadiri, M., 2007. Computer simulation of the effect of contact stiffness and adhesion on the fluidization behaviour of powders. Chem. Eng. Sci. 62, 184-194.

Motlagh, A.H.A., Grace, J.R., Salcudean, M., Hrenya, C.M., 2014. New structure-based model for Eulerian simulation of hydrodynamics in gas-solid fluidized beds of Geldart group "A" particles. Chem. Eng. Sci. 120, 22-36.

Muller, C.R., Holland, D.J., Sederman, A.J., Scott, S.A., Dennis, J.S., Gladden, L.F., 2008. Granular temperature: Comparison of Magnetic Resonance measurements with Discrete Element Model simulations. Powder Technol. 184, 241-253. 
Murray, J.A., Benyahia, S., Metzger, P., Hrenya, C.M., 2012. Continuum representation of a continuous size distribution of particles engaged in rapid granular flow. Phys. Fluids 24.

Ojha, R., Menon, N., Durian, D.J., 2000. Hysteresis and packing in gas-fluidized beds. Phys. Rev. E 62, $4442-4445$.

Pandit, J.K., Wang, X.S., Rhodes, M.J., 2006. On Geldart Group A behaviour in fluidized beds with and without cohesive interparticle forces: A DEM study. Powder Technol. 164, 130-138.

Patankar, S.V., 1980. Numerical heat transfer and fluid flow. Hemisphere Pub. Corp. McGraw-Hill, Washington, New York.

Quintanilla, M.A.S., Valverde, J.M., Castellanos, A., Viturro, R.E., 2001. Looking for self-organized critical behavior in avalanches of slightly cohesive powders. Phys. Rev. Lett. 8719, 194301.

Rabinovich, Y.I., Adler, J.J., Ata, A., Singh, R.K., Moudgil, B.M., 2000a. Adhesion between nanoscale rough surfaces - I. Role of asperity geometry. J. Colloid Interface Sci. 232, 10-16.

Rabinovich, Y.I., Adler, J.J., Ata, A., Singh, R.K., Moudgil, B.M., 2000b. Adhesion between nanoscale rough surfaces - II. Measurement and comparison with theory. J. Colloid Interface Sci. 232, 17-24.

Rhodes, M.J., 2008. Introduction to particle technology, 2nd ed. Wiley, Chichester.

Rhodes, M.J., Wang, X.S., Nguyen, M., Stewart, P., Liffman, K., 2001. Use of discrete element method simulation in studying fluidization characteristics: influence of interparticle force. Chem. Eng. Sci. 56, 69-76.

Rietema, K., Piepers, H.W., 1990. The effect of interparticle forces on the stability of gas-fluidized beds-I. Experimental evidence. Chem. Eng. Sci. 45, 1627-1639.

Royer, J.R., Evans, D.J., Oyarte, L., Guo, Q., Kapit, E., Mobius, M.E., Waitukaitis, S.R., Jaeger, H.M., 2009. High-speed tracking of rupture and clustering in freely falling granular streams. Nature 459, 11101113.

Sanchez-Delgado, S., Almendros-Ibanez, J.A., Garcia-Hernando, N., Santana, D., 2011. On the minimum fluidization velocity in 2D fluidized beds. Powder Technol. 207, 145-153.

Schroter, M., Goldman, D.I., Swinney, H.L., 2005. Stationary state volume fluctuations in a granular medium. Phys. Rev. E 71.

Silbert, L.E., Ertas, D., Grest, G.S., Halsey, T.C., Levine, D., Plimpton, S.J., 2001. Granular flow down an inclined plane: Bagnold scaling and rheology. Phys. Rev. E 64.

Sundaresan, S., 2003. Instabilities in fluidized beds. Annu. Rev. Fluid Mech. 35, 63-88.

Syamlal, M., Rogers, W., O'Brien, T.J., 1993. MFIX Documentation Theory Guide. 
Timoshenko, S., 1934. Theory of elasticity, 1st ed. McGraw-Hill, New York,.

Tsinontides, S.C., Jackson, R., 1993. The mechanics of gas fluidized beds with an interval of stable fluidization. J. Fluid Mech. 255, 237-274.

Tsuji, Y., Kawaguchi, T., Tanaka, T., 1993. Discrete particle simulation of two-dimensional fluidized bed. Powder Technol. 77, 79-87.

Tsuji, Y., Tanaka, T., Ishida, T., 1992. Lagrangian numerical simulation of plug flow of cohesionless particles in a horizontal pipe. Powder Technol. 71, 239-250.

Valverde, J.M., Castellanos, A., 2006. Random loose packing of cohesive granular materials. Europhys. Lett. 75, 985-991.

Valverde, J.M., Castellanos, A., 2007. Types of gas fluidization of cohesive granular materials. Phys. Rev. E 75.

Valverde, J.M., Castellanos, A., Mills, P., Quintanilla, M.A.S., 2003. Effect of particle size and interparticle force on the fluidization behavior of gas-fluidized beds. Phys. Rev. E 67.

Valverde, J.M., Quintanilla, M.A.S., Castellanos, A., 2004. Jamming threshold of dry fine powders. Phys. Rev. Lett. 92.

van der Hoef, M.A., Annaland, M.V., Deen, N.G., Kuipers, J.A.M., 2008. Numerical simulation of dense gas-solid fluidized beds: A multiscale modeling strategy. Annu. Rev. Fluid Mech. 40, 47-70.

van der Hoef, M.A., Ye, M., van Sint Annaland, M., Andrews, A.T., Sundaresan, S., Kuipers, J.A.M., 2006. Multiscale Modeling of Gas-Fluidized Beds, in: Guy, B.M. (Ed.), Advances in Chemical Engineering. Academic Press.

Weber, M.W., Hoffman, D.K., Hrenya, C.M., 2004. Discrete-particle simulations of cohesive granular flow using a square-well potential. Granul. Matter 6, 239-254.

Weber, M.W., Hrenya, C.M., 2006. Square-well model for cohesion in fluidized beds. Chem. Eng. Sci. $61,4511-4527$.

Weber, M.W., Hrenya, C.M., 2007. Computational study of pressure-drop hysteresis in fluidized beds. Powder Technol. 177, 170-184.

Xu, Q., Orpe, A.V., Kudrolli, A., 2007. Lubrication effects on the flow of wet granular materials. Phys. Rev. E 76.

Yang, R.Y., Zou, R.P., Yu, A.B., 2003. Effect of material properties on the packing of fine particles. J. Appl. Phys. 94, 3025-3034. 
